UCID- -21510

DE89 001284

\title{
Raman Scattering in Crystals
}

\author{
David F. Edwards
}

September 30, 1988

\begin{abstract}
DISCLAIMER
This report was pripared as an account of work sponsored by an agency of the United States Government. Neither the United States Government nor any agency theroof, nor any of their cmployces, makes any warranty, express of implicd, of assurina any legal liabjlity of responsibility for the accuracy, completeness, or usefulness of any informatiun, apparatus, producl, or process disclosed, or represents that its use would not infringe privately owned rights. Reference berein to any specific commercial product, process, or service by trade aame, tradertatk, manufacturer, or otherwise does not nexessarily constitute or impiy its andorsement, recommendation, of favoring by the United States Government of any agency thereof. The views and opinions of authors expressed herein do not necessarily state or rellect those of the United States Government or any agency thereof.
\end{abstract}

\section{LAWRENCE LIVERMORE NATIONAL LABORATORY
University of California - Livermore, California - 94550} Avaitabfe from: National Technical Intormation Service - U.S. Department of Commerce
5285 Porl Royal Road * Springfield, VA 22161 - A02 - (Microfiche ADI) 


\section{Contents}

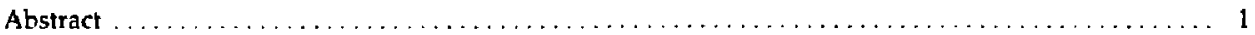

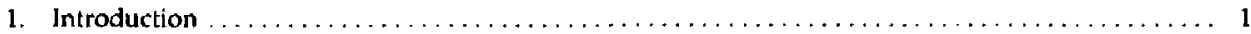

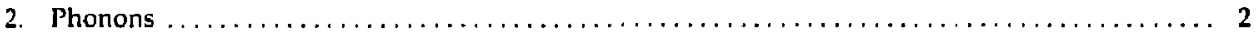

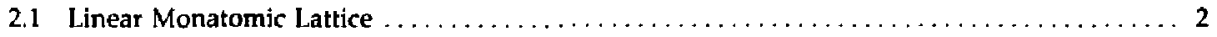

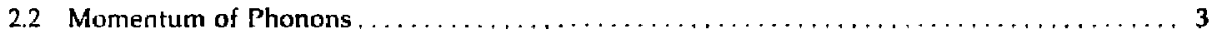

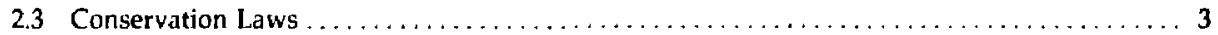

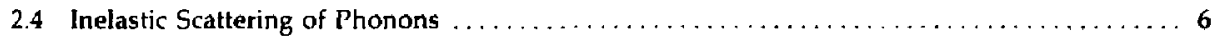

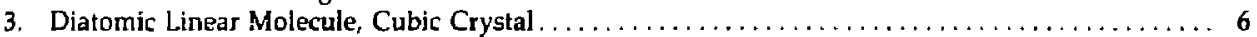

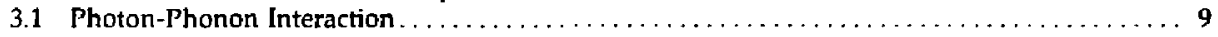

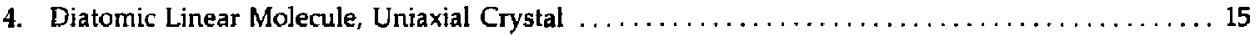

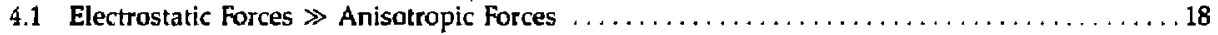

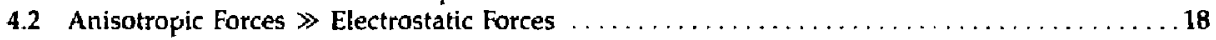

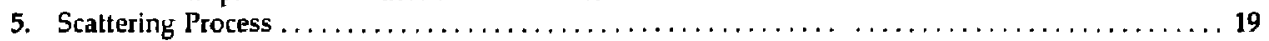

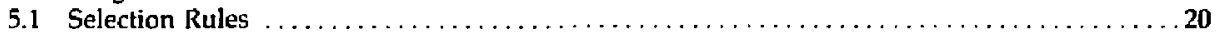

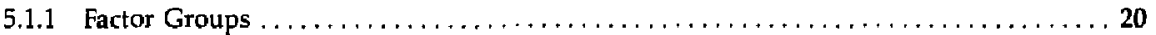

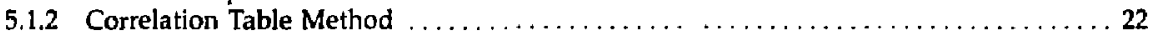

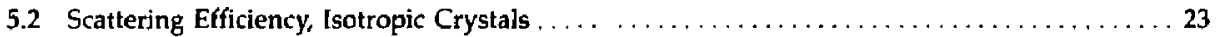

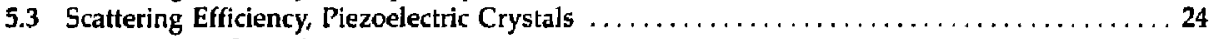

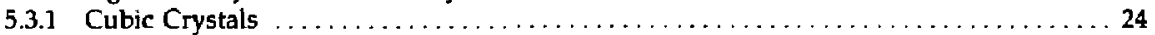

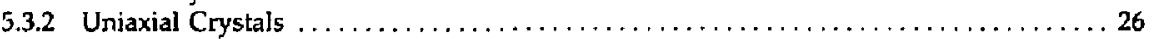

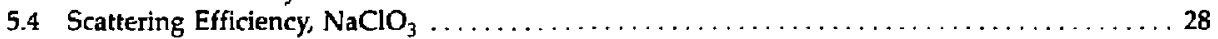

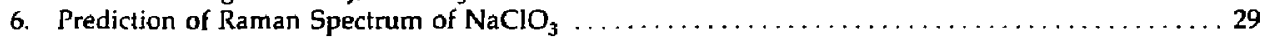

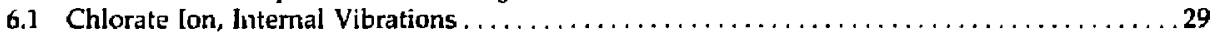

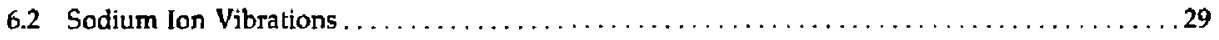

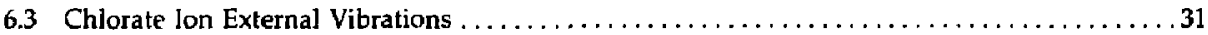

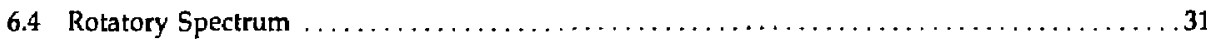

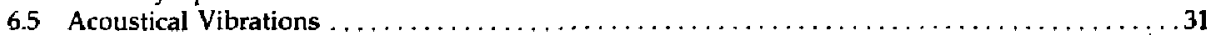

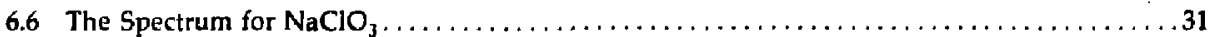

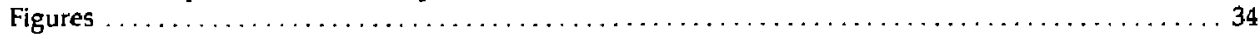

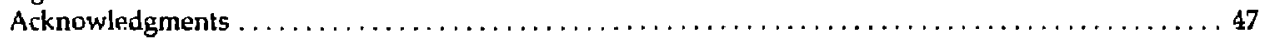

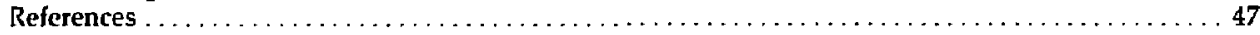




\title{
Raman Scattering in Crystals
}

\begin{abstract}
A tutorial presentation is given of Raman scattering in crystals. The physical concepts are emphasized rather than the detailed mathematical formalism. Starting with an introduction to the concepts of phonons and conservation laws, the effects of photonphonon interactions are presented. This interaction concept is shown for a simple rubic crystal and is extended to a uniaxial crystal. The correlation table method is used for determining the number and symmetry of the Raman active modes. Finally, examples are given to illustrate the relative ease of using this group theoretical method and the predictions are compared with measured Raman spectra.
\end{abstract}

\section{Introduction}

The purpose of this report is to provide a tutorial presentation of Raman scattering in crystals in which the physical concepts are emphasized rather than the detailed mathematical formalism. It is this writer's view that such a presentation is lacking in the literature and that the physical description should be instructive for those unfamiliar with Raman spectroscopy.

Since the invention of the $\mathrm{cw}$ gas laser, Raman scattering has experienced a great increase in popularity. Raman spectroscopy has now become a common tool in many laboratories. The high intensity and narrow linewidth of the laser output permit the measurement of the Raman scattered spectra of crystals under high resolution. Prior to the introduction of the laser the mercury discharge lamp was in common use as the Raman excitation source. With the mercury source many crystalline properties were either undetected or poorly resolved. Many of these problems have been rectified or reduced with the laser source. The collimated and plane-polarized characteristics of the laser output permit the nieasurement of the separate components of the scattered light facilitating the identification of the different vibrational modes in the crystal. Using the mercury source, positive identification of the different lattice modes was not always possible.

In writing this report it is assumed that the reader is familiar with solid state physics at least at the introductory level and has had a graduate level quantum mechanics course. The texts by $C$ Kittel, Introduction to Solid State Physics (John Wiley and Sons, New York, 3rd Edition) and by L. Schiff, Quantum Mechastics (McGraw-Hill Book Company, New York) are good general references and are about the 'evel of the material presented here. Additional reference material is listed in the bibliography. In preparing this report the writer has attempted to stress the physical concepts and to give sufficient detail and examples to illustrate the points being made. This approach has not always been taken in the past in texts and review articles on Raman scattering theory.

Given in Fig. 1 is a frequency-wave vector diagram illustrating the areas of applicability of the different scattering processes currently in vogue.' The coordinates are proportional to typical values of energy and momentum that are transferred during the different scattering processes illustrated. The approximate wave vector corresponding to the edge of the first Brillouin zone for a typical crystal would be approximately $1(\mathcal{A})$. . From this diagram it can be seen that optical experiments are generally along the $k=0$ axis and the neutron and $\gamma$-ray scattering experiments measure the crystalline properties at the zone edge. Shown in the neutron scattering region are typical data points from a scattering experiment on sodium. From the figure one can see large regions that are inaccessible by present scattering techniques. From this diagram it can be seen that a complete understanding of a crystal often requires measurements using several scattering techniques over a wide range of wave vector and frequency. Often these results must be combined with those of other measurements such as ultrasonic scattering to complement the scattering data. 
For a review of the different scattering techniques the reader is referred to the paper by Chu' and the references sited there. For the report presented here only Raman scattering will be discussed.

\section{Phonons}

By analogy with electromagnetic waves, the elastic waves in a solid are quantized and the quantum of energy is called the phonon. The earliest experimental evidence for the existence of these phonons or quantized lattice vibrations was the measurement of the heat capacity of solids at low temperatures. The inelastic scattering of $x$-rays and neutrons are also experimental evidence for quantized lattice vibrations. Before discussing several special properties of the phonon let us briefly restate the results found from the analysis of the vibrations of a linear monatomic lattice. ${ }^{2}$

\subsection{Linear Monatomic Lattice}

Consider a linear monatomic lattice. In general, the vibrations will be linear combinations of the vibrations parallel or perpendicular to the individual chains. Lattice waves with wave vector $k$ and vibrating perpendicular to the linear chain are called transverse phonons.

The equation of motion for an atom in plane $s$ is

$M \frac{d^{2} u_{s}}{d t^{2}}=\sum_{p} C_{r}\left(u_{i+p}-u_{s}\right)$,

where $u_{s}$ is the displacement of the atom of mass $M$ from its equilibrium position and $C_{p}$ is the force constant between planes $s$ and $p$. In general, $C_{p}$ will be different for transverse and longitudinal vibrations. Assuming a traveling wave solution of the form

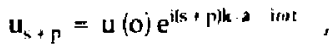

one finds

$\omega^{2}=\left(\frac{4}{M}\right) \sum_{p>0} C_{p, i} ; \sin 1 /\left.2 p k a\right|^{2}$

where $a$ is the lattice spacing. Restricting the interaction to nearest neighbors, $p=1$, we find

$\omega=\left(\frac{4 C_{1}}{M}\right)^{1 / 2}|\sin 1 / 2 \mathrm{ka}|$.

Equation (2.4) gives the relationship between $\omega$ and $k$, called the dispersion relation, with $C_{1}$, the force constant between adjacent atoms, as the constant of proportionality. Thus, in principle, Ior a monatomic lattice of atomic mass $M_{i}$ it is possible to evaluate the lattice force constant $C_{1}$ from the dispersion relation. Knowledge of the atomic interaction, $C_{1}$ is perhaps the most important property that one can obtain about the crystal. For a linear lattice of spacing a, the wave vector corresponding to the edge of the first Brillouin zone is $k_{\max }=\pi / a$. Typical values of the interatomic spacing are $a \sim 1 \AA$, thus $\mathrm{k}_{\max } \sim 10^{8} \mathrm{~cm}^{-1}$ or $1\left(\mathcal{A}^{-1}\right)^{-1}$ as shown in Fig. 1 .

For the Raman scattering experiments the wave vectors of interest are those near the center of the Brillouin zone, $k=0$. This is called the long wavelength limit. In the long wavelength limit, pka $\ll 1$ in Eq. (2.3) and

$w^{2}-k^{2} \frac{a^{2}}{M} \underset{n-1}{D} p^{2} C_{l}$ 
showing that the long range-forces, $C_{p}$, will become important because of the $\mathrm{p}^{2}$ term. We will see this to he the case when discussing the lattice dynamics of piezoelectric crystals.

\subsection{Momentum of Phonons}

A phonon having wave vector $k$ interacts with a photon or another phonon as if it had momentum hk. In reality a phonon on a lattice has physical momentum only when $k=0$. This can be seen as follows.

The linear momentum of the crystal can be witten as

$\mathrm{P}=\mathrm{m} \sum_{s=0}^{N-1} \dot{u}_{s}$,

where $m$ is the mass of the crystal. $m=M N^{2}$ for an $N$ by $N$ lattice of identical particles, each of mass $M$. The sum is over all atoms in N planes. Substituting from Eq. (2.2) we find

$p=-i \omega m u(0) e^{-i \omega t} \sum_{s=0}^{N-1} e^{i s k s}$

Rewriting the sum in Eq. (2,6), we find

$\sum_{s=0}^{N-1} e^{i s k s}=\frac{1-e^{i N k a}}{1-e^{i k a}}$.

For a crystal, the periodicity requires that $u_{z}=u_{q+N}$ or

$e^{\mathrm{iNka}}=1$,

which is just the periodic boundary condition.

Thus, from Eq. (2.6), $\mathrm{p}=0$, unless $\mathrm{k}=0$ and then

$P(k=0)=-i \omega M N^{3} u(0) e^{-i \omega t}$.

This means that the phonon carries zero momentum unless $k=0$. The reason for this can be seen from the following physical picture. Phonons are associated with the crystal and are essentially bound to it in contrast with a photon, which can exist independently of the crystal. The problem of interest, however, is the momentum transferred during a collision. For this case Beck ${ }^{3}$ has shown that the de Broglie relation, $\mathrm{p}=\hbar k$, is valid for phonons provided we give it a different interpretation. For elementary processes de Broglie's relation determines the momentum change of the total system when a phonon is emitted or absorbed; the total momentum of the system is not quantized in this case. The excitation of a phonon of wavelength $\lambda=2 \pi / \mathrm{k}$ implies a transfer of momentum $\mathrm{h} / \lambda$ to the motion of the center of gravity of the linear chain. The interatomic coordinates are relative coordinates and carry no momentum. The phonon behaves as if it had momentum $\hbar k$, sometimes called the crystal momentum, which is not the momentum in the ordinary sense. For more discussion of crystal momentum the reader is referred to the article by Beck ${ }^{3}$ or the texts by Kittel $^{2}$ or Shockley. ${ }^{4}$

\subsection{Conservation Laws}

The conservation conditions for Raman scattering can be derived classically by considering the incident electromagnetic wave interacting with the dielectric constant, which is undergoing thermal fluctuations. Such a derivation can be found elsewhere. ${ }^{5.6}$ The conservation of energy states

$\hbar \omega_{i} \pm \hbar \omega=\hbar \omega_{s}$, 
where $\omega_{i}, \omega$, and $\omega_{b}$ are the angular frequencies of the incident photon, the phonon, and the scattered photon, respectively. The conservation of momentum is

$\hbar \mathbf{k}_{\mathbf{i}} \pm \hbar \mathbf{k}=\hbar \mathbf{k}_{\mathrm{s}}$.

$\mathbf{k}_{i}, \mathbf{k}$, and $\mathbf{k}_{\mathrm{s}}$ are the wave vectors of the corresponding particles.

The conservation conditions can also be derived by analogy with the conditions for Brillouin scallering. Quantum mechanically, scattering from the thermally produced inhomogeneities is equivalent ${ }^{\prime}$ to the first-order Raman scattering associated with a transition in the vibrational states of the acoustic modes. Classically, the Brillouin scattering can be considered as the result of reflections of the electromagnetic waves from the traveling acoustic waves in the crystal. Because of interference effects the scattering will be directional dependent for a given wavelength acoustic wave. Also, the scattered light will be Doppler shifted because of motion of the acoustic wave. Following Heald, the conservation citiultions for Raman scattering will be obtained using this model for Brillouin scattering.

Let us consider the acoustic waves in the medium as a train of partially reflecting plane mirrors with spacing $\lambda=2 \pi / \mathrm{k}$ and moving with velocity v. A plane electromagnetic wave reflected from these surfaces will undergo a change in frequency due to the Doppler effect. Since the velocity of the mirror will add to that of the reflected wave, the direction of the reflected wave will be dependent upon v. Consider the case as shown in Fig. 2 . The incident electromagnetic wave is advancing with velocity $c$ and is reflected from the mirror advancing with velocity $v$. The effective interface is determined by Huygens principle. From geometrical cossiderations the interface between the incident and reflected wavefronts is defined from

$\mathrm{v} / \sin \gamma=\mathrm{c} / \sin \alpha$.

We wish to express these relationships in terms of the angles $\theta_{1}$ and $\theta_{2}$. This can be done when we note that the angles of incidence and reflection are equal relative to the effective interface and not relative to the mirror. Thus

$\theta_{1}+\gamma=\theta_{2}-\gamma$

Also $\theta_{1}+\theta_{2}+2 \alpha=\pi$, so that Eq. (2.8) becomes

$\frac{y}{c}=\frac{\sin 1 / 2\left(\theta_{2}-\theta_{1}\right)}{\cos \overline{1 / 2}\left(\theta_{2}+\theta_{1}\right)}$

The Doppler shift of the reflected wave can be obtained by considering two successive wave fronts incident on the moving mirror as shown in Fig. 3. From this, one finds

$\lambda_{1}=\ell \cos \theta_{1}, \lambda_{2}=\ell \cos \theta_{2}$,

or

$\ell=\frac{\lambda_{1}}{\cos \theta_{1}}=\frac{\lambda_{2}}{\cos \theta_{2}}$

Combining Eqs. (2.9) and (2.10) one obtains the Doppler-shifted frequency of the reflected wave. Writing $k=2 \pi / \lambda$. Eq. (2.10) becomes

$k_{1} \cos \theta_{1}=k_{2} \cos \theta_{2}$,

which are just the momentum components of the incident and reflected wave vectors paralle] to the mirror.

Shown in Fig. 4 are waves reflected from adjacent mirror surfaces. The Bragg condition for the incident wave is $n_{1} \lambda_{1}=\lambda_{p} \sin \theta_{1}$ and for the reflected wave $n_{2} \lambda_{2}=\lambda_{p} \sin \theta_{2}$. The order coefficients for 
both waves, $n_{1}$ and $n_{2}$, as well as their sum must be positive integers for interference to take place. Therefore

$\mathbf{n}=n_{1}+\mathbf{n}_{2}=\frac{\lambda_{\mathrm{p}} \sin \theta_{1}}{\lambda_{1}}+\frac{\lambda_{\mathrm{P}} \sin \theta_{2}}{\lambda_{2}}$

The electromagnetic properties of the crystal are approximately sinusoidally modulated by the acoustic waves, thus we can take $n=1$. Taking $k=2 \pi / \lambda$, Eq. (2.12) becomes

$\frac{2 \pi}{\lambda_{\mathrm{p}}}=\frac{2 \pi}{\lambda_{1}} \sin \theta_{1}+\frac{2 \pi}{\lambda_{2}} \sin \theta_{2}$

or

$k_{p}=k_{1} \sin \theta_{1}+k_{2} \sin \theta_{2}$.

which is just the perpendicular component of the momentum relation. Had we assumed the mirror moving in the opposite direction to that shown in Fig. 2 then $k_{p}$ would have a negative sign.

The energy conservation equation follows from $\mathrm{Eq} .(2.9)$

$\frac{\mathbf{v}}{\mathrm{c}}=\frac{\sin 1 / 2\left(\theta_{2}-\theta_{1}\right)}{\cos 1 / 2\left(\theta_{2}+\theta_{1}\right)} \cdot \frac{2 \sin 1 / 2\left(\theta_{2}+\theta_{1}\right)}{2 \sin 1 / 2\left(\theta_{2}+\theta_{1}\right)}=\frac{\cos \theta_{1}-\cos \theta_{2}}{\sin \left(\theta_{2}+\theta_{1}\right)}=\frac{\cos \theta_{1}-\cos \theta_{2}}{\sin \theta_{1} \cos \theta_{2}+\cos \theta_{1} \sin \theta_{2}}$.

Substituting $\cos \theta_{2}=\lambda_{2} / \lambda_{1} \cos \theta_{1}$ from Eq. (2.10) we find

$\frac{v}{c}=\frac{\lambda_{1}-\lambda_{2}}{\lambda_{2} \sin \theta_{1}+\lambda_{1} \sin \theta_{2}}$.

From Eq. (2.12) with $n=1$ we find

$\frac{\lambda_{1} \lambda_{2}}{\lambda_{\mathrm{p}}}=\lambda_{1} \sin \theta_{2}+\lambda_{2} \sin \theta_{1}$.

Substituting this expression into Eq. (2.14) we find

$\frac{v}{c}=\frac{\left(\lambda_{1}-\lambda_{2}\right) \lambda_{p}}{\lambda_{1} \lambda_{2}}$

or

$\frac{v}{\lambda_{\mathrm{p}}}=\frac{c}{\lambda_{2}}-\frac{c}{\lambda_{1}}$

$\omega_{\mathrm{p}}=\omega_{2}-\omega_{1}$,

where the sign of $\omega_{\mathrm{p}}$ depends on the direction of mirror motion. Equations (2.11), (2.13), and (2.15) are just the conservation equations

$\hbar\left(\omega_{1} \pm \hbar \omega_{p}=\hbar \omega_{2}\right.$,

and

$\hbar k_{1} \pm \hbar k_{\mathrm{p}}=\hbar k_{2}$.

Retaining $n \neq 1$ in Eq. (2.12) corresponds to multiphonon interaction, which are not of interest here. 


\subsection{Inelastic Scattering of Phonons}

For photon-phonon interaction, the energy on the photon is much greater than that of the phonon,

$\omega_{1 \text { or } 2} \gg \omega_{\mathrm{p}}$

or since $\omega_{1}=c k_{i}$ and $\omega_{p}=v k_{p}$, we have that $\left|k_{1} i-\right| k_{2} \mid$ and $\omega_{1} \sim \omega_{2}$.

It therefore follows that

$\mathbf{k}_{\mathrm{p}}=2 \mathrm{k}_{1} \sin \psi / 2$

or

$\omega_{\mathrm{p}}=\frac{2 \mathrm{v}_{\mathrm{s}} \omega \mathrm{n}}{\mathrm{c}} \sin \psi / 2$

where $\psi$ is the scattering angle; $v_{\mathrm{s}}$ and $\mathrm{n}$ are the velocity of sound and index of refraction in the crystal. A very important consequence of Eqs. (2.18) and (2.19) are that for a given scattering experiment with $k_{1}, \psi$, $v_{s}, \omega$, and $n$ specified, the wave vector and frequency, $k_{p}$ and $\omega_{p}$ of the phonon are completely specified. These formulas are true for Brillouin as well as Raman scattering.

Typical values for Brillouin scattering in solids ${ }^{9}$ are phonon frequencies of about $10^{9} \mathrm{~Hz}$ (energies of the order of $19^{-6} \mathrm{eV}$ ) and phonon wa:e vectors about $10^{4} \mathrm{~cm}^{-1}\left(10^{-4} \AA^{-1}\right)$.

For right-angle Raman scattering using visible lighi excitation $\left(20,000 \mathrm{~cm}^{-1}\right)$ the phonon wave vector and frequency inside the crystal are typically

$\left.k_{p} \sim\left(2 \pi \times n \times 2 \times 10^{4} \mathrm{~cm}^{-1}\right) \times \sqrt{2}=3 \times 10^{5} \mathrm{~cm}^{-1} \sim 10^{-3}(A)\right)^{-1}$

and

$\omega=k_{p} v_{5}=3 \times 10^{5} \times 5 \times 10^{5} \sim 2 \times 10^{11} \mathrm{~Hz}\left(10^{-3} \mathrm{eV}\right)$.

Referring to Fig. 1 it can be seen that these values of phonon frequency and wave vector fall within the appropriate areas of the figure for Raman and Brillouin scattering. In deriving Eq. (2.18) it was assumed that the fractional change in the magnitude of the photon wave vector was small. For the two examples given above this fractional change is about $10^{-5}$ for the two scattering processes, thus justifying the assumption that

$\left|k_{1}\right| \sim\left|k_{2}\right|$.

\section{Diatomic Linear Molecule, Cubic Crystal}

It will be instructive to consider in some detail a crystal with two atoms per unit cell. By considenng the interaction of the lattice vibrations of this crystal with the electromagnetic radiation, we can gain insight into the expected Raman spectra of the crystal and show how these spectra might change under certain perturbations. We will first consider a cubic crystal for which the forces are isotropic. In Sec, 4, the uniaxial crystalline problem will be considered.

Let us consider the cubic lattice as shown in Fig. 5. The mass of the atoms are taken as $M_{1}$ and $M_{2}$, respectively. The lattice spacing is $2 \mathrm{a}$. If we assume nearest neighbor interaction only, then the equations of motion can be written as

$\left.\begin{array}{l}M_{1} \frac{d^{2} u_{2 s+1}}{d t^{2}}=C\left(u_{2 s+2}+u_{2 s}-2 u_{2 s+1}\right) \\ \text { and } \\ M_{2} \frac{d^{2} u_{2 s}}{d t^{2}}=C\left(u_{2,+1}+u_{2 s, 1}-2 u_{2 s}\right)\end{array}\right\}$ 
We vish the solutions for the two types of atoms to be of the form of traveling waves having different amplitude,

$u_{2 s+1}=\xi \mathrm{e}^{\mathrm{i}|12 s+1| k, 1} \quad$ (ut|

and

$u_{2 s}=\eta \mathrm{e}^{\mathrm{i} \mid 2 \mathrm{~s} k \mathrm{a}-\omega) \mid}$

Substituting these into the equations of motion and imposing the periodic boundary condition we find

$\omega^{2}=C\left(\frac{1}{M_{1}}+\frac{1}{M_{2}}\right) \pm C\left[\left(\frac{1}{M_{1}}+\frac{1}{M_{2}}\right)^{2}-\frac{4 \sin ^{2} k a}{M_{1} M_{2}}\right]^{1 / 2}$.

This solution is shown in Fig. 6 for the case $M_{2}>M_{1}$.

The values of $\mathrm{k}$ can lie any place within the first Brillouin zone. For typical values of the lattice constant the maximum wave vector will be of the order

$\mathrm{k}_{\max } \sim 3 \times 10^{8} \mathrm{~cm}^{-1}$

From Sec. 2 and Fig. 1, we estimated

$k \sim 3 \times 10^{5} \mathrm{~cm}^{-1}<k_{\max }$

for right-angle Raman scattering. Therefore, ka $\sim 10^{-2}$ and Eq. (3.3) can be approximated by the following for $k \rightarrow 0$,

$\omega_{+}^{2} \sim 2 C\left(\frac{1}{M_{1}}+\frac{1}{M_{2}}\right)$ (independent of $k$ )

and

$\omega_{-}^{2} \sim \frac{2 C}{M_{1}+M_{2}} k^{2} a^{2},(\omega \rightarrow k)$.

The upper branch, corresponding to the plus sign in Eq. (3.3), is called the optical branch because we can show that for transverse displacements of the particles at $k=0$ (i.e., the long wavelength limit) the two atoms vibrate against each other with a fixed center of mass. To show this we substitute the assumed solutions (3.2) into the equations of motion (3.1) and for $k=0$ find

$-\omega^{2} \mathrm{M}_{1} \xi=2 \mathrm{C} \eta-2 \mathrm{C} \xi$

$-\omega^{2} M_{2} \eta=2 C \xi-2 C \eta$.

Dividing one of these equations by the other, we have

$\left(\frac{M_{1}}{M_{2}}\right) \frac{\xi}{\eta}=\frac{\eta-\xi}{-(\eta-\xi)}$

or

$\xi=-\left(\frac{\mathbf{M}_{2}}{\mathbf{M}_{1}}\right) \eta$. 
This shows that the two atoms of masses $M_{1}$ and $M_{2}$ move in the opposite directions. If these atoms carried charges of opposite sign, this type of vibration could be excited by an optical field and hence the name optical branch. More about this branch later.

The lower branch corresponding to the minus sign in Eq. (3.3) is called the acoustical branch and has the character of being linearly proportional to $k$ for small $k$ values as seen by Eq. (3.4). The phase velocity corresponding to this lower branch is

$v=\frac{\omega}{k}=\left(\frac{2 C}{M_{1}+M_{2}}\right)^{1 / 2}$ a ,

for the long wavelength limit.

Consider Jongitudinal vibrations in an elastic string. The phase velocity is given by ${ }^{2}$

$\mathrm{v}=(\mathrm{E} / \rho)^{1 / 2}$,

where $E$ is Young's modulus and $\rho$ is the linear density. For the diatomic lattice

$\mathbf{E}=\mathrm{aC}$

and

$\rho=\frac{M_{1}+M_{2}}{2 a}$

Substituting these values into Eq. (3.9) we find the phase velocity in the elastic string to be

$v=\left(\frac{2 C}{M_{1}+M_{2}}\right)^{1 / 2} a$

which is identical with Eq. (3.8),

The amplitude ratio $\xi / \eta$ for these acoustic vibrations is

$\xi / \eta=\frac{2}{2-M_{1}\left\{\left(\frac{1}{M_{1}}+\frac{1}{M_{2}}\right)-\left[\left(\frac{1}{M_{i}}+\frac{1}{M_{2}}\right)^{2}-\frac{4 \operatorname{sir}^{2} k a}{M_{1}}\right]_{2}^{1 / 2}\right]}$

from Eq. (3.5) and $\omega^{2}$ from Eq. (3.4) corresponding to the acoustic vibrations. In the limit of small $\mathbf{k}$ we have $\xi / \eta \rightarrow 1$, which means the two masses $M_{1}$ and $M_{2}$ are moving in unison, the same as adjacent masses in the elastic string. For this reason the mode is called the acoustic mode and the lower branch of Fig. 6 is the acoustic branch.

In general, electromagnetic waves propagating within the crystal will interact only with lattice vibrations of the same wavelength and same approximate frequency. From the order of magnitude estimates above we saw that for right-angle Raman scattering $k \sim 3 \times 10^{5} \mathrm{~cm}^{-1}<k_{\max }$. This means that for Raman scattering we are in the long wavelength limit; i.e., small $k$ values. From Eqs. (3.3) (3.5), and (3.6) we see that for $\mathrm{k} \rightarrow 0$

$\omega_{\text {acous }} \rightarrow \mathbf{0}$

but $\omega_{\text {opt }}$ remains finite. In the discussion of the optical behavior of a crystal we will therefore only be concerned with the optical branch.

The above discussion can be extended to the case of more than two atoms per unit cell. For $p$ atoms in the primitive cell there will be 3 p equations of motion. Three equations will correspond to the acoustical 
modes; unifurm undistorted translation of the cell as a whole at $k=0(\omega=0)$. The remaining $3(p-1)$ modes will each have a nonzero frequency in the long wavelength limit and are the optical modes. For the case of $p=2$ ( $\mathrm{NaCl}$, for example) the three optical branches can be classified approximately into one longitudinal and two transverse branches in the long wavelength limit. For the interactions considered so far these three branches will be degenerate. By considesing the long range electrostatic interaction origir.ating from the longitudinal motion of the ions, we will show that this degeneracy is partially removed.

Physicully, one can see this by considering the transverse and longitudinal motions of adjacent atoms for the optical branch as shown in Fig. 7. We have seen above that the acoustical branch is not important to our problem. For the transverse motion of the optical branch (TO) the net dipole moment produced by the ionic displacements will be zero because the adjacent particles have opposite charges and will cancel each other. For the longitudinal motion of the optical branch (LO) there will be a net dipole moment produced by the ionic displacements in both time and space. Thus, for the longitudinal motion there will be an electrostatic interaction in addition to the elastic restoring force previously considered. For the transverse motion there will only be the elastic forces In the long wavelength limit the frequency of the longitudinal motion will be greater than for the transverse motion, $\omega$ (LO) $>\omega$ (TO). This photonphonon interaction is discussed in greater detail in the following section.

The elastic force interaction, also called the deformation potential interaction, is proportional to the relative displacement of the positive and negative sublattices during vibration. The long range electromagnetic interaction is proportional to the electric field produced by the induced dipole moment. For ionic crystals the electric moment associated with the vibration is strong so that the electromagnetic interaction is important.

\subsection{Photon-Phonon Interaction}

The macroscopic theory of long wavelength optical vibrations for a diatomic molecule has been given by Huang. ${ }^{3,19}$ For describing the optical vibrations, the most convenient parameter specifying the relative displacement of the positive and negative ions is the reduced mass,

$M=\frac{M_{1} M_{2}}{M_{1}} \cdot \frac{M_{2}}{M_{2}}$

and the roordinate

$w=\left(u^{+}-u^{-}\right) /(M n)^{1 / 2}$,

where $u^{+}-u^{-}$is the relative displacement of the positive and negative ionic lattices and $\mathbf{n}$ is the number of cells per unit volume.

The equations of motion describing the diatomic molecule, including, the deformation potential and electrostatic interactions, can be obtained from the Lagrangian density ${ }^{11}$

$\mathscr{L}=\frac{1}{2} \dot{w}^{2} k-\left(\frac{1}{2} \gamma_{11} w^{2}-\gamma_{12} w \cdot E-\frac{1}{2} \gamma_{22} E^{2}\right)$.

where the $\gamma$ 's are undetermined constants and $\mathrm{E}$ is the macroscopic internal electric field arising from the induced dipole moment. The equation of motion is

$\ddot{w}+\gamma_{11} w-\gamma_{12} E=0$.

The momentum density conjugate to $w$ is $\partial \mathscr{g} \partial \dot{w}=\dot{w}$, and the Hamiltonian density, $\mathscr{X}=\mathrm{T}+\mathrm{V}$, is

$\mathscr{H}^{\prime}=\frac{1}{2} \dot{w}^{2}+\frac{1}{2} \gamma_{11} w^{2}=\gamma_{12} w \cdot E-\frac{1}{2} \gamma_{22} E^{2}$. 
The polarization, given by

$\mathbf{P}=\frac{\partial . \not{H}}{\partial \mathrm{E}}=\gamma_{12} w+\gamma_{22} \mathbf{E}$

is the macroscopic dielectric polarization in terms of the displacement and electric field vectors, $w$ and $E$. The equation of motion shuws that in addition to the usual Hooke's law restoring force there is an electromagnetic restoring force. The polarization equation is made up of a lattice polarization proportional to the relative displacement and an electric field induced term. Assuming plane wave solutions, the equations become

$\mathbf{w}\left(\gamma_{11}-\omega^{2}\right)=\gamma_{12} E$

$\mathbf{P}=\gamma_{12} \mathbf{w}+\gamma_{22} \mathbf{E}$

Following the usual procedures ${ }^{11}$ we solve Eq. (3.11) in terms of the experimentally measurab!e quantities $\varepsilon_{0}, \varepsilon_{x}$, and $\omega_{1}$, the dielectric constants at low and optical frequencies, respectively, and the lattice transverse vibration frequency. The important result is that first derived by Lyddane, Sachs, and Teller (LST); that is

$\omega_{\xi}=\left(\frac{\varepsilon_{0}}{\varepsilon_{x}}\right)^{1 / 2} \omega_{\mathrm{t}}$

In general $\varepsilon_{x}<\varepsilon_{0}$ so that $\omega_{8}>\omega_{1}$ in the long wavelength limit. The degeneracy between the one longitudinal and the two transverse vibrations has been removed. Shown in Fig. 8 is the long wavelength region of the dispersion curve. For the scale used only the optical frequencies $\omega_{\ell}(L O)$ and $\omega_{1}$ (TO) are indicated. The acoustical frequencies would coincide with the $k$-axis.

The $\gamma$-coefficients in Eq. (3.11) are found ${ }^{13}$ to be the following:

$$
\begin{aligned}
& \gamma_{13}=\omega_{1}^{2} \\
& \gamma_{12}=\left(\frac{\varepsilon_{0}-\varepsilon_{\infty}}{4 \pi}\right)^{1 / 2} \omega_{\mathrm{t}} \\
& \gamma_{22}=\frac{\varepsilon_{\infty}-1}{4 \pi} .
\end{aligned}
$$

Substituting these parameters into Eq. (3.11) we find

$\mathbf{P}=\left(\frac{\varepsilon_{0}-\varepsilon_{*}}{4 \pi}\right)^{\mathrm{t} / 2} \omega_{1} w+\frac{\varepsilon_{x}-1}{4 \pi} E$

and

$w\left(\omega_{1}^{2}-\omega^{2}\right)=\left(\frac{\varepsilon_{0}-\varepsilon_{x}}{4 \pi}\right)^{1 / 2} \omega_{1} E$

The lattice dynamiss presented so far are properties of the diatomic crystal assuming no external effects. The dispersion relation for an electromagnetic field inside the crystal is just

$\omega=\frac{c}{\sqrt{\varepsilon_{x}}} k$, 
and is shown in Fig. 8. The phase velocity $c / \sqrt{\epsilon_{\infty}}=2 \times 10^{10} \mathrm{~cm} / \mathrm{sec}$ is just the slope of the dotted line.

In the region of the crossover of the photon and phonon dispersion curves the interaction between the phonons $\left(\omega_{p}\right.$ and $\left.\omega_{1}\right)$ and the photon becomes very important. Physically, the transverse lattice vibrations mix with the electromagnetic field to form the observed infrared dispersion. The interaction between ions has no effect on the longitudinal lattice motion so $\omega_{l}$ will be unchanged. The effects of the phonon-photon interaction can be seen from the coupled solutions of the lattice dynamics equations (3.12) and (3.13) and Maxwell's equations.

Assuming plane wave solutions of the form

$\mathbf{P}=\mathbf{P}_{\mathbf{0}} \mathrm{e}^{\mathrm{i}(\mathbf{k} \cdot \mathbf{R}-\boldsymbol{\omega} t)}$

$\mathbf{w}=\mathbf{w}_{0} \mathrm{e}^{\mathrm{i}(\mathbf{k} \cdot \mathbf{R} \cdots \omega t)}$

$E=E_{0} e^{i(k \cdot R-t u t)}$,

Maxwell's equations can be written as

$\mathbf{E}=\frac{-4 \pi\left[\mathbf{k}(\mathbf{k} \cdot \mathbf{P})-\omega^{2} \mathbf{P} / c^{2}\right]}{k^{2}-\omega^{2} / c^{2}}$

For the transverse vibrations $\mathbf{k} \cdot \mathbf{P}=0$ and the solution of Eqs. (3.12), (3.13), and (3.14) is

$\omega^{2}=\frac{1}{2 \varepsilon_{x}}\left(\omega_{\mathrm{t}}^{2} \varepsilon_{0}+c^{2} k^{2}\right) \pm\left[\frac{1}{4 \varepsilon_{\infty}^{2}}\left(\omega_{1}^{2} \varepsilon_{0}+c^{2} k^{2}\right)^{2}-\omega_{\mathrm{t}}^{2} k^{2} c^{2} / \varepsilon_{\infty}\right]^{1 / 2}$

or

$\frac{k^{2} c^{2}}{\omega^{2}}=\frac{\varepsilon_{0} \omega_{\mathrm{t}}^{2}-\varepsilon_{x} \omega^{2}}{\omega_{\mathrm{t}}^{2}-\omega^{2}}$

In the long wavelength limit, $k \rightarrow 0$, the solutions to Eq. (3.15) become

$\omega^{2}=\omega_{\mathrm{t}}^{2}\left(\varepsilon_{0} / \varepsilon_{\infty}\right)$

and

$\omega^{2}=\left(c^{2} / \varepsilon_{\infty}\right) k^{2}$

For the longitudinal vibrations $\mathbf{k} \cdot \mathbf{P}=\mathrm{kP}$ and

$\omega=\omega_{\mathrm{t}}\left(\frac{\varepsilon_{0}}{\varepsilon_{\infty}}\right)^{1 / 2}=\omega_{p}$

Equation (3.18) is just the LST relation we found earlier for the case of no coupling between the phonon and photon, which shows that the ionic interaction has no effect on the longitudinal vibrations.

Shown in Fig. 9 are the solutions (3.15) and (3.18) for a hypothetical crystal, using the frequency and dielectric coefficient values of $\mathrm{NaCl}$. The longitudinal vibration (LO) is nondegenerate but the transverse vibrations (TO) are two-fold degenerate reflecting the two independent orientations of $\mathbf{E}$ in the plane normal to $\mathbf{k}$.

At this point it will be instructive to digress momentarily to derive the dispersion relation for the frequency dependence of the dielectric coefficient. To do this we must first generalize the equations of motion by introducing a damping term. This can be done by adding to the Lagrangian density, $\mathscr{L}_{x}$ the Rayleigh dissipative function ${ }^{12}$ 
$F=\frac{1}{2} \gamma \dot{w}^{2}$

which is the usual ad hoc way of introducing the dissipative term. When this is done, in place of Eq. (3.13), we have

$\mathbf{w}\left(\omega_{\mathrm{t}}^{2}-\omega^{2}+\mathrm{i} \gamma \omega\right)=\left(\frac{\varepsilon_{0}-\epsilon_{*}}{4 \pi}\right)^{1 / 2} \omega_{\mathrm{t}} \mathbf{E}$

where $\gamma$ is the damping coefficient. Substituting this expression for $w$ into Eq. (3.12) we find

$\mathbf{P}=\frac{1}{4 \pi}\left(\varepsilon_{x}-1+\frac{\left(\varepsilon_{0}-\varepsilon_{x}\right) \omega_{1}^{2}}{\left(\omega_{t}^{2}-\omega^{2}\right)+i \omega \gamma}\right) \mathbf{E}$.

The dielectric coefficient is

$\varepsilon(\omega)=1+4 \pi \mathrm{P} / \mathrm{E}$

or

$\varepsilon[\omega]=\varepsilon_{x}+\frac{\mathrm{S}}{\omega_{1}^{2}-\omega^{2}+\mathrm{i} \gamma \omega}$.

where $S=\left(\epsilon_{0}-\epsilon_{x}\right) \omega_{t}^{2}$ is a dimensionless mode strength. Eq. (3.19) shows the dispersion relation for the dielectric coefficient.

Returning to Fig. 9, the transverse optical frequency, $\omega_{1}$, can be measured by several methods, one of which is absorption spectroscopy. For linear polarized radiation normal to a thin plate, the transmission will be a minimum at $\omega=\omega_{1}$ as shown below.

Assuming the incident light propagating along the $x$ axis, the following relations are valid for the scalar magnitudes of the electric field.

incident side $\left(A \mathrm{e}^{\mathrm{i} \omega \times / \epsilon}+B \mathrm{e}^{-\mathrm{i \omega x} / \mathrm{g}}\right) \mathrm{e}^{-\mathrm{i \omega t}}$

within the plate $\left(C \mathrm{e}^{\mathrm{i} \omega \mathrm{\omega} \times / c}+D \mathrm{e}^{-i \omega n \times / c}\right) \mathrm{e}^{-\mathrm{i} \omega t}$

far side $E \mathrm{e}^{\mathrm{i}(\omega \times / \epsilon \cdot \omega t)}$.

Included in the expressions are the reflected components of the electric field and $\mathrm{r}$ is the refractive index of the crystal. Imposing the usual boundary conditions that the field be continuous at $x=0$ and $x=d$, the amplitude ratio for the incident and far side fields is given by

$\frac{E}{A}=\frac{4 n e^{-i / \omega d / c}}{\left[(n+1)^{2} e^{i \omega n d / r}-(n-1)^{2} e^{i \omega n d / c}\right]}$.

For thin plates $\omega d / c \ll 1$ the ratio becomes

$$
\frac{E}{A}=\frac{1}{1-i \frac{\omega d}{2 c}\left(n^{2}+1\right)} \text {. }
$$

The transmittance is just the magnitude squared of this ratio and is written as

$$
T=\left|\frac{E}{A}\right|^{2}=\frac{1}{1+i \frac{\omega d}{2 c}\left(n^{* 2}-n^{2}\right)}=\frac{1}{1+i \frac{\omega d}{2 c}\left(\varepsilon^{*}-\varepsilon\right)} .
$$


For very thin plates $T$ will be a minimum when $\omega\left(\varepsilon^{*}-\varepsilon\right)=\omega\left(n^{* 2}-n^{2}\right)$ is a maximum. From the dispersion relation $\mathrm{Eq}$. (3.19) we have

$\omega\left(\varepsilon^{*}-\varepsilon\right)=\frac{i S \omega^{2} \gamma}{\left(\omega_{1}^{2}-\omega^{2}\right)^{2}+(\omega \gamma)^{2}}$

The maximum condition is found from

$\frac{\mathrm{d}}{\mathrm{d} \omega}\left[\frac{\omega^{2}}{\left(\omega_{\mathrm{t}}^{2}-\omega^{2}\right)^{2}+\omega^{2} \gamma^{2}}\right]=0$

The solution is $\omega=\omega_{1}$ and corresponds to the minimum in the transmission for thin plates. $\omega_{t}$ is the frequency at which the refractive index, $n$, and the dielectric constant, $\varepsilon$, become very large.

For the hypothetical crystal ciescribed in Fig. 9 the value of $\omega_{\mathrm{t}}=3.09 \times 10^{13} \mathrm{~Hz}$ is that measured by Barnes ${ }^{13}$ for the thin sodium chloride crystal. For polar crystals there are several methods for finding the longitudinal optic mode frequency, $\omega_{p}$.

The first method depends upon the application of the LST relation, provided the low and optical frequency dielectric constants, $\varepsilon_{0}$ and $\varepsilon_{\infty}$, are known. From the given data $\omega_{\ell}$ is found from the LST relation

$\omega_{p}=\omega_{\mathrm{t}}\left(\frac{\varepsilon_{0}}{\varepsilon_{x}}\right)^{1 / 2}$

The value shown in Fig. 9, $\omega_{p}=4.6 \times 10^{13} \mathrm{~Hz}$ was obtained using the data $\varepsilon_{0}=5.62$ and $\varepsilon_{\infty}=2.25$. This method is reasonably reliable for ionic crystals but for crystals with small energy gaps or more than one reststrahlen band there will be uncertainty in the $\varepsilon_{0}$ and $\varepsilon_{\infty}$ values.

A second method is to identify $\omega_{2}$ and $\omega_{Q}$ with the lower and upper frequencies of the reststrahlen band of the crystal. The theory ${ }^{7}$ behind this is as follows. For normal incidence, the reflectance is given by

$R(\omega)=\left|\frac{[\varepsilon(\omega)]^{1 / 2}-1}{|\varepsilon(\omega)|^{1 / 2}+1}\right|^{2}$,

where $\varepsilon(\omega)$ is the frequency dependent dielectric coefficient given by Eq. (3.19). As $\omega$ is increased from zero to $\omega_{1}$, the value of $E(\omega)$ increases to a large value with the effect that $R=1$. For frequencies slightly larger than $\omega_{t}, \varepsilon$ switches from a positive to a negatively large value up to the frequency corresponding to

$\varepsilon(\omega)=\varepsilon_{x}+\frac{S}{\omega_{1}^{2}-\omega^{2}+i \gamma \omega}=\varepsilon_{\infty}+\frac{\left(\varepsilon_{0}-\varepsilon_{\infty}\right) \omega_{1}^{2}}{\omega_{i}^{2}-\omega^{2}+i \gamma \omega}=0$.

The solution is

$\omega=\omega_{1}\left(\frac{\varepsilon_{0}}{\varepsilon_{x}}\right)^{1 / 2} \equiv \omega_{1}$

which is just the LST relation. For frequencies $\omega_{\mathrm{q}}<\omega<\omega_{\rho}$ the dielectric constant is negative so that the complex index of refraction $n=\sqrt{\varepsilon}$ is pure imaginary. The dielectric constant can be written

$\varepsilon(\omega)=(\mathbf{n}-\mathbf{i} \delta)^{2}$,

where $\boldsymbol{n}$ is the refractive index and $\delta$ is the extinction coefficient. Within the crystal, for a pure imaginary index of refraction, the planar waves will be of the form of damped exponential waves with the damping coefficient proportional to $\delta$. Therefore, for frequencies $\omega_{t} \leq \omega \leq \omega_{p}$, the reflectivity will be large and the 
crystal will be highly absorbing. Curve fitting of the measured reflectance data using the dielectric dispersion relation with $\omega_{\mathrm{r}}$ as an adjustable parameter is a second method of determining the longitudinal optical frequency.

Other methods have been used for determining $\omega_{t}$ and $\omega_{p}$. Each has its particular advantages and disadvantages. It is not the purpose of this paper to discuss these further but the interested reader should refer to the literature ${ }^{\text {I4 }}$ for details.

In conclusion to this section, we have shown that the photons and phonons mix in the presence of electromagnetic radiation within the crystal. The transverse lattice vibrations mix with the radiation field and together they become the observed infrased dispersion. This mixture of photons and transverse optical phonons in a polar crystal is called a "polariton." A number of papers ${ }^{15}$ have appeared recently describing the theoretical and experimental properties of polaritons. Details of these properties will not be given here but one can see that they are an extension of the present discussion.

In Fig. 9 those waves with $\omega<\omega_{\mathrm{t}}$ are predominantly mechanical oscillations of the lattice (phononlike) with only a small fraction of their energy being radiative. Ignoring the mixing for these waves, we have just the transverse vibrations of Fig. 8. For these vibrations the phase velocity is small compared with $c / \sqrt{\varepsilon_{x}}$ and the electrostatic approximation is adequate. For those transverse vibrations with $\omega>\omega_{p}$ their phase velocities are greater than $c / \sqrt{\varepsilon_{\infty}}$ and the electrostatic approximation is invalid. For these vibrations the mixing is so large that the vibrations are photon-like. In Fig. 9 for $\omega \leq 2 \omega_{1}$ and $k \leq 2 \omega_{1}\left(\epsilon_{0}\right)^{1 / 2} c$ the upper and lower vibrations are phonon- and photon-like, respectively.

For the scale used in Fig. 9 the acoustic branch coincides with the absissia. For example, at $\mathbf{k}=$ $5 \times 10^{4} \mathrm{~cm}^{-1}$ the frequency of the acoustic mode will be about $4 \times 10^{10} \mathrm{~Hz}$ and would be indistinguishable from the $k$-axis of Fig. 9.

The conservation conditions of Eqs. (2.16) and (2.17) can be used to determine the frequency at which a Raman phonon will be exrited. Rewriting the conservalion conditions.

$\omega_{i}-\omega_{s}=\omega$

$\mathbf{k}_{\mathbf{i}}-\mathbf{k}_{\mathrm{s}}=\mathbf{k}$

and using

$k=\frac{\omega}{v}$ and $k_{s}=\frac{\omega_{s}}{\eta_{s}}$.

it is possible to eliminate $\omega_{5}$ from Eqs. (3.20) and (3.21). $v_{j}$ and $v_{5}$ are the phase velocities of the incident and Raman scattered radiations. Squaring Eq. (3.21) and making the above substitution one finds

$k^{2}=\frac{\omega_{i}^{2}}{v_{i}^{2}}+\frac{\omega_{s}^{2}}{v_{s}^{2}}-2 \frac{\omega_{i} \omega_{s}}{v_{i} v_{s}} \cos \theta$.

where $\theta$ is the angle between $\mathbf{k}_{i}$ and $\mathbf{k}_{5}$. Using Eq. (3.20) to eliminate $\omega_{s^{\prime}}$, the above equation becomes

$\omega=\omega_{i}\left(1-\frac{v_{s}}{v_{i}} \cos \theta\right) \pm\left[v_{s}^{\frac{z}{t}} k^{2}-\omega_{i}^{2} \frac{v_{s}^{2}}{v_{i}^{2}} \sin ^{2} \theta\right]^{1 / 2}$.

For the dispersion curve, such as Fig. 9, Eq. (3.22) specifies the allowed phonon frequencies, $\omega$, for a given phonon wave vector, $\mathbf{k}$ (magnitude $|\mathbf{k}|$ and direction $\theta$ ).

For forward scattering $(\theta=0)$ the phonon frequency is given by

$\omega=\omega_{i}\left(1-\frac{v_{s}}{v_{i}}\right)+v_{s} k$. 
For an isotropic crystal $v_{s}=v_{i}$, then Eq. (3.23) becomes

$\omega=v_{s} \mathbf{k}$.

This expression is just the dispersion curve for the laser photon of frequency $\omega_{5}$ and is shown as the dotdashed line in Fig. 9. In general, $v_{s}>v_{\mathrm{t}}$ since the index of refraction increases with frequency so that for an isotropic crystal with $\theta=0$ the photon dispersion curve will intersect the photon dispersion curve as shown by the dot-dashed curve marked $\theta=0, v_{5}>v_{j}$. The intersection of these two curves specifies the values of $(\omega, \mathrm{k})$, which will occur. Polaritons are produced at the crossing of these two lines. From Fig. 9 one can thus see a range of polariton frequencies are available by varying the scattering angle. Experimentally, this has been shown to be the case for the cubic crystal GaP. ${ }^{16}$

Right-angle Raman scattering is of more interest to the present problem. The phonon frequency, obtained from Eq. (3.22) with $\theta=\pi / 2$, becomes

$\omega=\omega_{i} \pm\left[v_{s}^{2} k^{2}-\omega_{i}^{2} \frac{v_{s}^{2}}{v_{i}^{2}}\right]^{1 / 2}$.

With $i_{s}=v_{i}$ the wave vector intercept $(\omega=0)$ corresponds to

$\mathrm{k}=4.1 \times 10^{5} \mathrm{~cm}^{-1}$

for the crystal of Fig. 9. The $\theta=\pi / 2$ curve of Eg. (3.25) is shown in the broken scale section at the right side of Fig. 9. For the parameter values used, the right-angle Raman line will be vertical indicating a TO and an LO phonon will be involved in the Raman scattering. The edge of the Brillouin zone is at about $10^{8} \mathrm{~cm}^{-1}$, which lies off the right-hand edge of Fig. 9 emphasizing the interest in the long wavelength limit.

From Fig. 9 one can see that, assuming $\omega_{l}$ and $\omega_{1}$ correspond to Raman-active vibrations, the Raman spectra for the diatomic cubic crystal will consist of two lines at frequencies $\omega_{p}$ and $\omega_{l}$. The transverse vibration will be doubly degenerate.

Mooradian et al. ${ }^{17}$ have reported the measurements of the Raman spectra for several polar cubic crystals having two atoms per unit cell. The zincblende semiconductor crystals, GaAs, InP, AlSb, and GaP are examples of such crystals and their data are reproduced in Fig. 10. For each crystal only two Raman active modes are measured; a twofold degenerate transverse mode and a singly degenerate longitudinal mode. For these polar cubic crystals the electrostatic interaction removes the degeneracy between the TO and $L O$ vibrations. For the materials measured this separation between $\omega_{p}$ and $\omega_{\mathrm{t}}$ was from about $21 \mathrm{~cm}^{-1}$ for AlSb to about $44 \mathrm{~cm}^{-1}$ for InP.

It is interesting to compare the values of $\omega_{P}(\mathrm{LO})$ and $\omega_{1}$ (TO) measured from the Raman spectrum with those values found from reflection spectra. Johnson ${ }^{18}$ measured $\omega_{p}(\mathrm{LO})=294 \mathrm{~cm}^{-1}$ and $\omega_{t}(\mathrm{TO})=$ $266 \mathrm{~cm}^{-1}$ from the reflection spectrum of GaAs, which are in good agreement with the values $\omega_{\rho}(\mathrm{LO})=$ $284 \mathrm{~cm}^{-1}$ and $\omega_{1}$ (TO) $=261 \mathrm{~cm}^{-1}$ measured by Mooradian et al. ${ }^{7}$ The Raman data are probably more exact because of inaccuracies ${ }^{14}$ in the dispersion relation as mentioned previously.

\section{Diatomic Linear Molecule, Uniaxial Crystal}

The long wavelength lattice vibration properties for uniaxial crystals can be obtained from a generalization of the analysis for the cubic crystal of Sec. 3.

In a uniaxial crystal the light propagation properties are described by two indices of refraction. Imagine a point source of light within our crystal. At any instant of time the wavefront forms a continuously expanding surface. For a uniaxial crystal this surface will be either a sphere or an ellipsoid of revolution depending on the polarization of the light. The sphere and ellipsoid will touch along a certain axis defined to be the optic axis (also called the c-or z-axis) of the crystal. Due to this anisotropy of the crystal, the vibrational frequencies of the atoms moving parallel to the c-axis will be different than when the motion is in the plane perpendicular to the c-axis. We will call these $\omega_{\models}$ and $\omega_{\perp}$, respectively. The 
static and optical frequency dielectric constants will also be different for these directions; $\varepsilon_{\|}, \varepsilon_{1}^{\prime \prime}, \varepsilon_{1}$, and $\varepsilon_{\perp}^{0}$. The phonon spectrum can be obtained from expressions similar to Eqs. (3.10), (3.11), and (3.12) for the parallel and perpendicular components of $\mathbf{W}, \mathbf{E}$, and $\mathbf{P}$. There will be six equations for the six unknowns $\omega_{\|}, \omega_{1}, E_{\|}, E_{1}, P_{\|}$, and $P_{1}$. We will have a nontrivial solution only if the determinant of the coefficients vanishes. This determinant is shown on the facing page. In the determinant $\theta$ is the angle between the propagation vector $\mathbf{k}$ and the c-axis and $\alpha$ is the angle between the displacement vector $\mathbf{D}$, and the plane defined by the $\mathbf{c}$-axis and $\mathbf{k}$. For ordinary waves $\mathbf{D} \perp \mathbf{k}$, so that $\cos \alpha-0$. Fö the $\epsilon_{\text {intrast }}$ uiillary wave $\mathbf{D}$ and $\mathbf{k}$ are coplanar so that $\cos \alpha=1$.

Rather than solving for the general case, the solutions corresponding to ordinary and extraordinary waves are of most interest. For the ordinary wave $\mathbf{E}$ and $\mathbf{P}$ are perpendicular to both $\mathbf{k}$ and the $\mathbf{c}$-axis $(\cos \alpha=0)$ and Eq. (4.1) becomes

$\omega^{4} \varepsilon_{\perp}-\omega^{2}\left[c^{2} k^{2}+\omega_{\perp}^{2} \varepsilon_{1}^{0}\right]+c^{2} k^{2} \omega_{\perp}^{2}=0$,

or

$\frac{\mathrm{k}^{2} \mathrm{c}^{2}}{\omega^{2}}=\frac{\varepsilon_{\perp}^{0} \omega_{\perp}^{2}-\varepsilon_{\perp} \omega^{2}}{\omega_{\perp}^{2}-\omega^{2}}$ (ordinary wave) ,

which ase the same forms as Eq. (3.15) for the cubic crystal. For the extraorainary wave $D \perp k$, but $E$ and $P$ do not, in general, have a simple orientation relative to $k$ or the $c$-axis $(\cos \alpha=1)$. The solution to Eq. (4.1) for the extraordinary wave becomes

$$
\frac{k^{2} c^{2}}{\omega^{2}}=\frac{\left(\omega_{\|}^{2} \varepsilon_{\|}^{0}-\omega^{2} \varepsilon_{\|}\right)\left(\omega_{\perp}^{2} \varepsilon_{\perp}^{0}-\omega^{2} \varepsilon_{\perp}\right)}{\left(\omega_{\|}^{2}-\omega^{2}\right)\left(\omega_{\perp}^{2} \varepsilon_{\perp}^{0}-\omega^{2} \varepsilon_{\perp}\right) \sin ^{2} \theta+\left(\omega_{\perp}^{2}-\omega^{2}\right)\left(\omega_{\|}^{2} \varepsilon_{\|}^{0}-\omega^{2} \varepsilon_{\|}\right) \cos ^{2} \theta}
$$

(extraordinary wave) .

For the special case of $\theta=0$, that is, propagating along the optic axis, the extraordinary wave reduces to the ordinary wave; Eq. (4.3) transforms into Eq. (4.2).

In the long wavelength limit $(k=0)$, Eq. (4.2) becomes

$\omega^{2}=\omega_{\perp}^{2}\left(\frac{\varepsilon_{\perp}^{0}}{\varepsilon_{\perp}}\right)$ (ordinary wave) ,

and from Eq. (4.3)

$\omega^{2}=\omega_{\|}^{2}\left(\frac{\varepsilon_{\|}^{\|}}{\varepsilon_{\|}}\right)=\left(\omega_{\|}^{p}\right)^{2} \quad$ (extraordinary wave)

and

$\omega^{2}=\omega_{\perp}^{2}\left(\frac{\varepsilon_{\perp}^{0}}{\varepsilon_{\perp}}\right)=\left(\omega_{\perp}^{0}\right)^{2}$

The expressions (4.5) and (4.6) define $\omega_{f}^{f}$ and $\omega_{\perp}^{\ell}$ analogous to $\omega_{\ell}$ defined by Eq. (3.16). In the long wavelength limit the spectra will consist of a doubly degenerate line at $\omega=\omega_{\perp}^{\ell}$ and a singly degenerate line ar $\omega=\omega_{\|}^{f}$ as given by Eqs. (4.4)-(4.6).

For right-angle Raman scattering $\mathrm{k} \sim 10^{5} \mathrm{~cm}^{-1}$ and $\omega / \mathrm{c} \sim 1 \mathrm{~cm}-1$ so that $\mathrm{k}^{2} \mathrm{c}^{2} / \omega^{2} \rightarrow \infty$. From Eqs. (4.2) and (4.3) this means

$\omega=\omega, \quad$ (ordinary wave) 

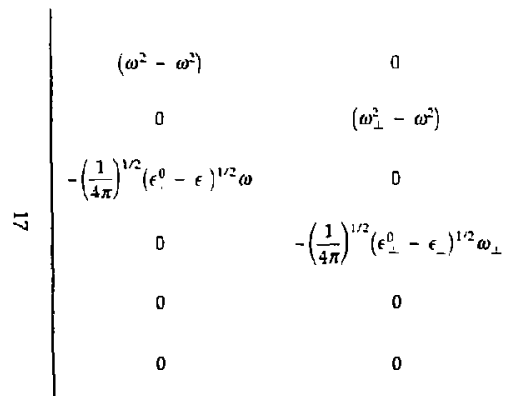

$-\left(\frac{1}{4 \pi}\right)^{1 / 2}\left(\epsilon^{0}-\epsilon\right)^{1 / 2} \omega$ $-\left(\frac{1}{4 \pi}\right)^{1 / 2}\left(\epsilon_{\perp}^{0}-\epsilon_{\perp}\right)^{1 / 2} \omega_{\perp}$

0

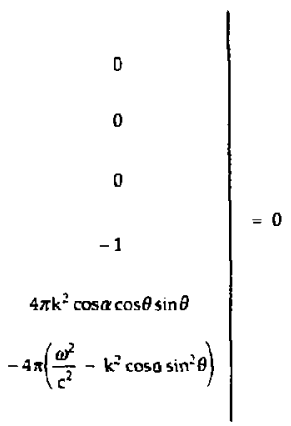


and

$\frac{\left(\omega^{2} \varepsilon_{1}^{0}-\omega^{2} \varepsilon_{1}\right)}{\left(\omega^{2}-\omega^{2}\right)} \cos ^{2} \theta+\frac{\left(\omega_{\perp}^{2} \varepsilon_{\perp}^{0}-\omega^{2} \varepsilon_{1}\right)}{\left(\omega_{\perp}^{2}-\omega^{2}\right)} \sin ^{2} \theta=0 \quad$ (extraordinary wave) .

Thus, the Raman spectrum will be expected to have an angle $\theta$ independent line corresponding to the ordinary wave Eq. (4.7) and an angulas dependent extraordinary Raman spectrum as shown in Eq. (4.8). For $\theta=0$ and $\theta=\pi / 2$ the spectrum for the extraordinary wave will be a doubly degenerate line with frequency given by Eqs. (4.5) and (4.6), respectively. For the general direction, the Raman spertrum will consist of three lines, two of which will have angular dependent frequencies.

Dispersion curves can be drawn for the uniaxial crystal similar to those shown in fig. 9 . For the uniaxial crystal we will consider two limiting cases depending on the relative magnitudes of the anisotropic forces and the electrostatic forces.

\subsection{Electrostatic Forces $\gg$ Anisotropic Forces}

The electrostatic forces remove the degeneracy of the longitudinal and transverse vibrations as shown in Fig. 8. The anisotropic forces arise because of the birefringence of the uniaxial crystal. For a negative uniaxial crystal $\omega_{i}^{\prime}>\omega_{\perp}^{\prime}$ and $\omega_{j}^{\rho}>\omega_{\perp}^{p}$. The opposite is true for a pesitive uniaxial crystal. For the dominant electrostatic forces case we have

$$
\omega-\omega_{\perp} \mid \ll \omega_{l}^{\ell}-\omega_{i}^{\mathrm{t}} \text { and } \omega_{\perp}^{\mathrm{q}}-\omega_{i}^{\mathrm{i}}
$$

Hexagonal $\mathrm{ZnO}$ is a positive uniaxial crysta! for which Eq. (4.9) is satisfied. ${ }^{19}$ Figure 11 is a schematic of the dispersion relations Eqs. $[(4.2)$ and (4.3)] for the propagation vector parallel $(\theta=0)$ and perpendicular $(\theta=\pi / 2)$ to the $c$-axis corresponding to this limiting case for a positive uniaxial crystal such as $\mathrm{ZnO}$. For a general value of $\theta$, one root of Eq. (4.3) will lie in the vicinity of $\omega_{4}^{t}$ and $\omega_{2}^{t}$, while the other root will be close to $\omega_{\perp}^{\mathrm{t}}$ and $\omega$ ?

The Raman spectrum for a positive uniaxial crystal, such as is shown in Fig. 11, would be expected to have two lines for $\theta=0$ and three for $\theta=\pi / 2$. For $\theta=0$, the lines would correspond to the (2) $\omega_{\perp}^{2}$ and (1) $\omega_{i}^{p}$ vibrations and for $\theta=\pi / 2$ the lines would correspond to the $\omega_{\perp}^{t}, \omega_{l}^{t}$ and $\omega_{\perp}^{p}$ vibrations. For a giver measurement the separations $\left|\omega_{l}^{t}-\omega_{1}^{t}\right|$ and $\left|\omega_{l}^{p}-\omega_{1}^{f}\right|$ may be too small to resolve the angular dependence predicted by Eq. (4.3).

\subsection{Anisotropic Forces $\gg$ Electrostatic Forces}

The anisotropic forces are mainly responsible for the separations $\left|\omega_{l}^{\ell}-\omega_{i}^{\ell}\right|$ and $\left|\omega_{i}^{l}-\omega_{\perp}^{l}\right|$. The electrostatic forces produce the separations $\left|\omega_{l}^{\ell}-\omega^{l}\right|$ and $\left|\omega_{\perp}^{\ell}-\omega_{\perp}^{t}\right|$. For the limiting case of interest here, the anisotropic forces are much greater than the electrostatic forces so that

$$
\omega^{p}-\omega_{-}^{t}|\gg| \omega^{p}-\omega^{t} ! \text { and }\left|\omega_{-1}^{p}-\omega_{-}^{t}\right| \text {. }
$$

The solutions of Eq. (4.3) for these approximations will be

$\omega^{2}=\left(\omega_{i}^{2}\right)^{2} \sin ^{2} \theta+\left(\omega_{1}^{f}\right)^{2} \cos ^{2} \theta$

and

$\omega^{2}=\left(\omega_{\perp}^{\prime}\right)^{2} \cos ^{2} \theta+\left(\omega^{P}\right)^{2} \sin ^{2} \theta$. 
The dispersion curves shown in Fig. 12 correspond to a positive uniaxial crystal with the anisotropic forces dominating the electrostatic forces. As the angle between the propagation vector and the r-axis is increased from 0 to $\pi / 2$ the upper Raman line will change from transverse extraordinary to longitudinal extraordinary while the lower branch changes from a longitudinal extraordinary at $\theta=0$ to an ordinaryextraordinary degenerate transverse vibration at $\theta=\pi / 2$. On physical principles one would expect a large anisotropic effect for this crystal because only a transversely polarized phonon can be infrared active.

She et al. ${ }^{21}$ have shown that quartz is an example of a crystal for which the inequality, Eq. (4.10), is satisfied. The angular dependence predicted by Eqs. (4.11) and (4.12) as given by She et al. is reproduced in Fig. 13. Here one can see for certain vibrations the variation of the phonon frequencies with phonon propagation direction.

\section{Scatiering Process}

The microscopic description of the Raman scattering process can he expressed quantum mechanically in several different ways ${ }^{21}$ depending upon the decomposition of the Hamiltonian of the system. For the Raman process one photon is annihilated from the incident beam, one Raman photon is created and one phonon is created; the number of photons and phonons differ by 3 in the initial and final states. By this description the Raman effect is called a three-particle process. For a three-particle process the vector potential operators for the two photons and one phonon app:ar each time in the matrix element of the scattering matrix describing the process. This means that the lowest order nonzero scattering matrix will correspond to third-order perturbation theory. The third-order perturbation calculations are tedious and the details can be found elsewhere. ${ }^{21,22}$ The conservation of momentum and energy Eqs. (2.7a-b) follow directly from this calculation with the essential result that the Raman scattering efficiency for isotropic crystals is given by

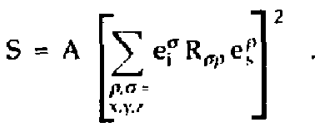

$\mathbf{e}_{\mathrm{i}}^{\sigma}$ and $\mathbf{e}_{\mathrm{s}}^{\prime \gamma}$ are components of the unit vectors $\hat{\mathrm{e}}_{\mathrm{i}}$ and $\hat{\mathrm{e}}_{\mathrm{s}}$ in the polarization directions of the incident and scattered photons. $A$ is a constant of proportionality and $R_{a p}$ is a second rank tensor called the Raman tensor. The subscripts on $R_{\sigma f}$ indicate the photon polarization directions. The form of the Raman tensor $R_{\sigma p}$ is as follows:

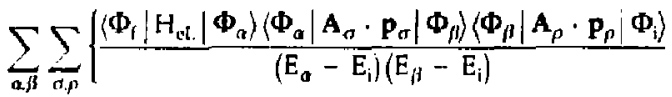

$$
\begin{aligned}
& +\frac{\left\langle\Phi_{\mathrm{f}}\left|\mathbf{A}_{\sigma} \cdot \mathbf{P}_{\sigma}\right| \Phi_{\mathrm{a}}\right\rangle\left\langle\Phi_{a}\left|H_{\mathrm{el} L}\right| \Phi_{\beta}\right\rangle\left\langle\Phi_{\beta}\left|A_{\beta} \cdot \mathbf{p}_{p}\right| \Phi_{\mathrm{i}}\right)}{\left(\mathrm{E}_{\alpha}-\mathrm{E}_{\mathrm{i}}\right)\left(\mathrm{E}_{\beta}-\mathrm{E}_{\mathrm{i}}\right)}
\end{aligned}
$$

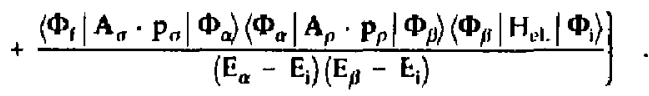

This is the sum of three terms each of which contains the product of two matrix elements involving photon annihilation and creation operators (for example, $\left\langle\Phi_{\alpha}\left|\mathbf{A}_{\sigma} \cdot \mathbf{P}_{\sigma}\right| \boldsymbol{\Phi}_{\beta}\right)$ and $\left\langle\Phi_{\beta j}\left|\boldsymbol{A}_{\rho} \cdot \mathbf{P}_{\rho}\right| \Phi_{\mathrm{i}}\right)$ ) and one matrix element involving the phonon annihilation and creation operators (for example, $\left(\Phi_{f}\left|H_{e l}\right| \Phi_{a}\right)$ ). In the above expression $\left.\mid \Phi_{i}\right)$ and $\left.\mid \Phi_{j}\right)$ are the initial and final state functions and $\left.\mid \Phi_{a}\right)$ and $\left|\Phi_{j}\right\rangle$ are intermediate state functions. The energy denominations are defined in the appendix. $\mathrm{H}_{\mathrm{et}}$ corresponds to the electron-lattice interaction Hamiltonian and contains both phonon annihilation and creation operators. The electric potential operators, $\mathbf{A}_{1}$, contain photon annihilation and creation operators. 
The Feynman diagrams corresponding to Eq. (5.2) will each have three interaction vertices, one corresponding to each matrix element. There will be six of these diagrams because each matrix element in Eq. (5.2) contains two operators; an annihilation and a creation operator. The six Feynman diagrams are shown in Fig. 14. The first diagram corresponds to the first term in Eq. (5.2). In this figure, the first interaction vertix corresponds to an incident photon being annihilated from the initial state function $\left(\boldsymbol{\Phi}_{i}\right)$. The second interaction is that of a Raman-photon creation involving the intermediate states. The third interaction involves a phonon creation and the final state function $\left.\mid \Phi_{1}\right)$. Interchanging the order of the photon annihilation and creation produces the second Feynman diagram of Fig. 14. Following this same procedure we can show the origin of the six diagrams of Fig. 14.

Ovander ${ }^{23}$ has made a group-theoretical determination of the different Raman tensors corresponding to each of the 32 crystal classes. A summary of this calculation is given in the appendix. Collected in Table I are the symmetry species (irreducible representations) corresponding to the allowed Raman transitions. In the table, $x, y$, or $z$ in brackets after an irreducible representation iridicates that the vibration is infrared artive and has the direction of polarization indicated. As an example, the Raman-active $A$ vibration of crystal class $C_{3}$ is infrared active with polarization along the $z$-axis. In Table $I$, directly above the irreducible representation, is a matrix, which gives the nonvanishing components of the Raman tensor. The different elements of the matrices are the nine components of the tensor obtained by allowing both $\sigma$ and $\rho$ to take on the values $x, y$, and $z$.

\subsection{Selection Rules}

The selection rules for the Raman eifect in ionic crystals can be found with the application of simple group theoretical methods. Given below is a brief discussion of the physical concepts of the method and the details can be found in the literature $e^{24-27}$ and standard texts ${ }^{28}$ on group theory. For the discussion given here it is assumed that the reader has at least an introductory knowledge of group theory including the meaning of chararter tables.

\subsubsection{Factor Groups}

The selection rules for the Raman effect in ionic crystals are obtained using the factor group analysis. A basic assumption of this analysis, ${ }^{26}$ also called the "unit cell approach," is that the intermolecular interactions are strong so that vibrational coupling exists ber ween molecules.

The unit cell is a basic building block of the crystal. The crystal lattice is constructed by successive translations of this unit cell. The unit cell here is defined as the smallest unit in which no atoms are equivalent under the translation operation. The atoms within the unit cell may be equivalent under other symmetry operations but translations are excluded. The symmetry of the crystal is one of the 230 space groups and each is an infinite group since they include translations. Mathematically, the space group of a crystal is that group of operations which carries an atom into an identical atom. The symmetry of the unit cell is described by a factor group of the space group operations and is called the unit cell group or factor group. The factor group is defined from the space group when the transiation operations are taken as identities. By this definition, the factor group will be finite. In general, the factor group is not a point group of the crystal but it is isomorphous with the 32 crystallographic point groups. ${ }^{27}$ In practice, the factor group is never derived from the space group but is always obtained from an inspection of the isomorphous point group. The isomorphous point group is easily obtained from the space group by deleting the superscript from the Schoenflies notation. For example, for a crystal with space group $\mathrm{C}_{2 \mathrm{~h}}^{5}$, the corresponding factor group will be isomorphous with the point group $C_{2 h}$ and the $C_{2 h}$ character table would be used as the character table for the factor group. The character table for the point group is identical with that for the factor group, however, some mirror planes and $n$-fold axes in the point group may become glide planes and screw axes in the factor group.

The correlation table method is a convenient method for applying the factor group analysis. Prerequisites for this method are knowledge of the crystal space group and site group symmetries, and the number of molecules per unit cell. Fateley et al. ${ }^{29}$ have compiled all the necessary tables for the application of the correlation method. This method is applied in Sec. 6 to determine the number and symmetry of the Raman vibrations in crystalline $\mathrm{NaClO}_{3}$. Given in this section will be only an outline of the method. 
Table I. Raman-active vibrational symmetries and Raman tensors.

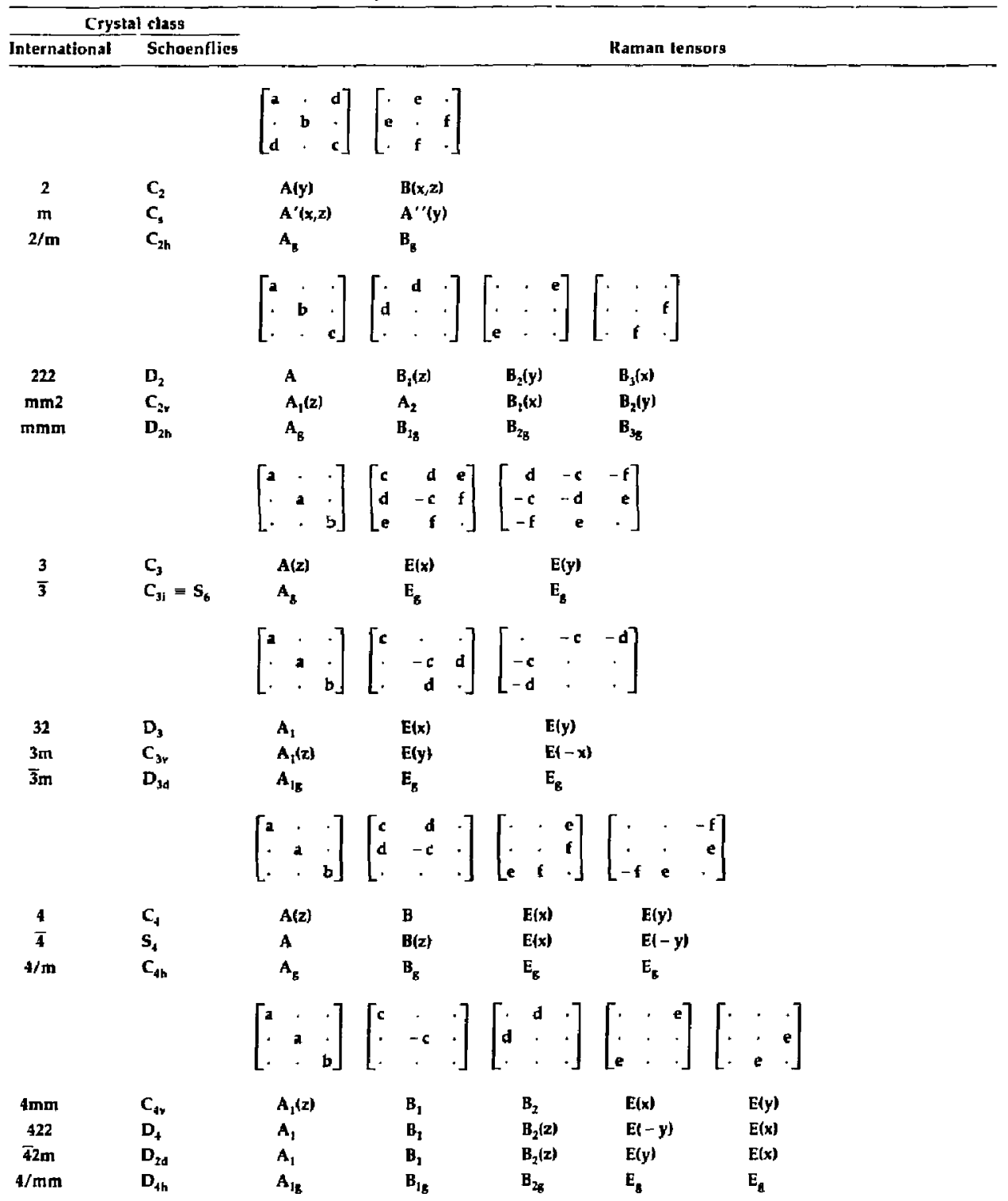


Table 1. (Continued).

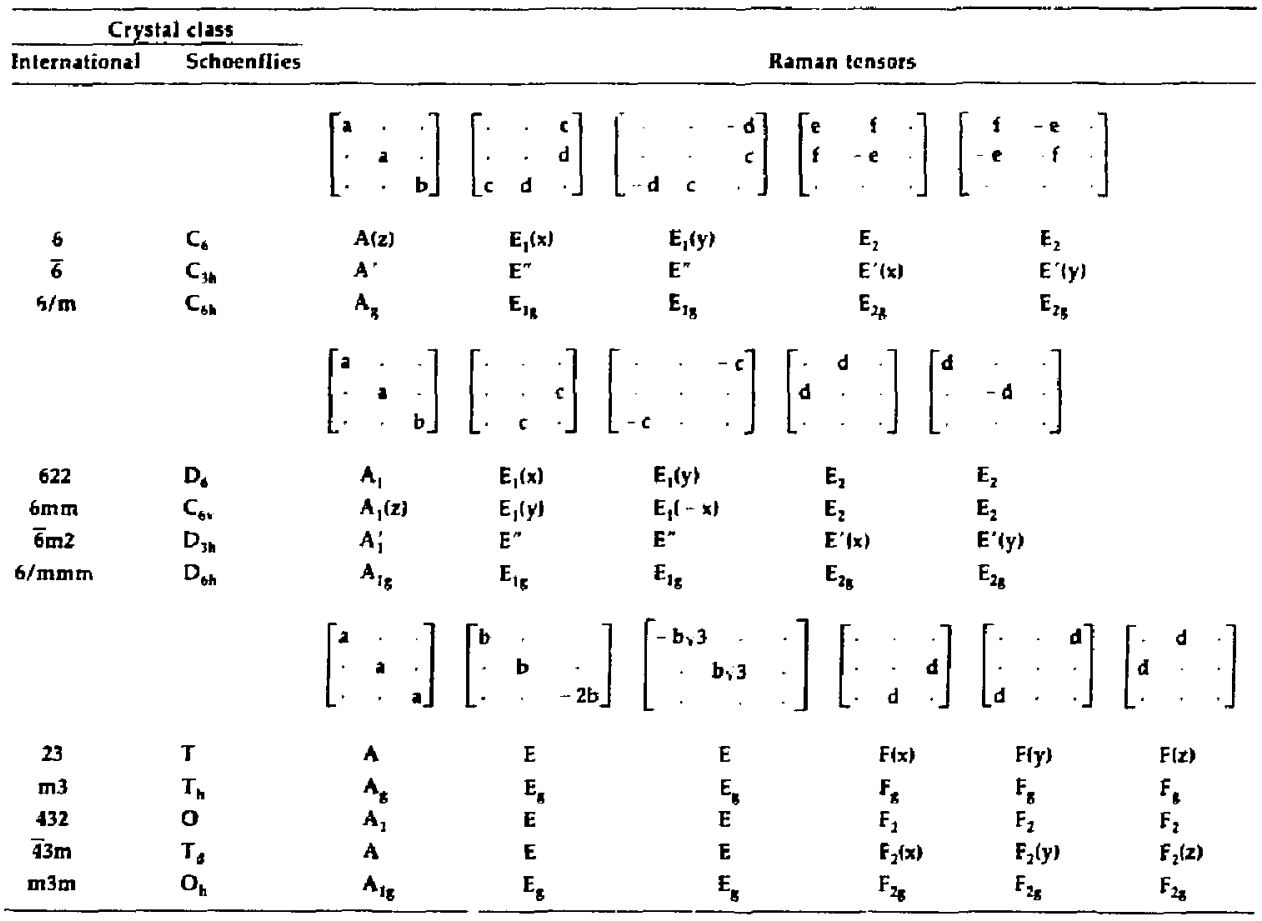

\subsubsection{Correlation Table Method ${ }^{24,29}$}

By way of review, the character table of the point group $C_{3 v}$ is given in Table II. The last two columns of the character table are particularly important for the factor group analysis given here. The translational unit vector transformations are indicated by $T_{x}, T_{y}$, and $T_{z}$ in the next to last column opposite their corresponding species. For example, in Table 11 , the translational unit vector parallel to tho crystallographic $z$ direction. $T_{z}$, has symmetry species $A_{1}$. The species of the three rotational unit vector transformations $R_{x}, R_{y}$ and $R_{z}$ are indicated opposite each vector. Infrared and Raman activities of the differest species are indicated in the last and next to last columns of the character table. A mode will be infrared active if it transforms as one or more of the translational unit vectors. In Table II species $A_{1}$ and $E$ will be infrared active. A mode will be Raman active if it transforms as ane or more of the direct products, $a_{x x}$ etc., of the translational unit vectors. The direct products are indicated in the last column of the character table. For example, in Table ll, species $A$ and $E$ will be Raman active.

For the application of the correlation method the unit cell may be considered to be made up of a combination of $\mathrm{p}$ polyatomic molecules or ions and $\mathrm{s}$ monatomic ions. Considering first the polyatomic molecules or ions, the number of modes and their symmetries are determined using the point group of the free molecule or ion multiplied by the number of polyatomic molecules and their number of internal degrees of freedom. The number of internal degrees of freedom for an $n$ atom molecule or ion is $(3 n-6)$, where we have subtracted three translational and three rotational external degrees of freedom. The symmetry species of these $2 \times(3 p)$ external degrees of freedom are the same as those of the transformations of the translational and rotational unit vectors given in the point group character table.

The next step is to correlate the point group symmetry species of the internal and extemal modes with the symmetry species of the site group of the polyatomic molecule or ion. Following this we correlate 
Table II. Character table for the point group $C_{3 v}$.

\begin{tabular}{|c|c|c|c|c|c|}
\hline$C_{3 v}$ & $\mathbf{E}$ & $2 \mathrm{C}_{3}$ & $3 n$ & & \\
\hline $\mathbf{A}_{1}$ & 1 & 1 & ] & $\mathrm{T}$ & $a_{x x}+a_{y y} \cdot a_{z z}$ \\
\hline A & 1 & I & -1 & $\mathbf{R}_{\mathbf{z}}$ & \\
\hline $\mathbf{E}$ & 2 & $-I$ & 0 & $\left(T_{x}, T_{y}\right) ;\left(R_{x}, R_{y}\right\}$ & $\left(a_{x y}-\alpha_{y y}, a_{x y}\right) ;\left(a_{y z}, a_{2 x}\right)$ \\
\hline
\end{tabular}

the site group symmetry species with the symmetry species of the factor group. The correlation of one species under one symmetry to a species under another symmetry can be determined by excmining the character tables of the two species or by using published correlation tables. ${ }^{29,30}$

The $s$ monatomic atoms or ions in the crystal wili give rise to $3 \mathrm{~s}$ translational modes. Correlation in this case is between the site group of the ions and the crystal factor group. The site symmetry species are the same as those of the translational unit vector transformations in the character table of the site group.

Iacluded within the correlation of the external modes will be three translational degrees of freedom corresponding to the acoustic modes. As pointed out in Sec. 2, these three degroes of freedom correspond

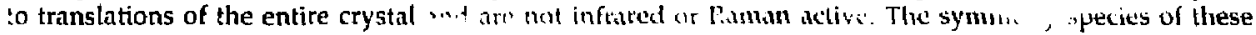
acoustic modes are the same as the transformations of the translational unit vectors in the factor group character table. These acoustic modes are subtracted from the other modes of the same species leaving only the genuine translatory lattice modes.

The infrared and Raman activity of the internal and external modes under the factor group symmetry are determined form an examination of the character table as described above.

The factor group analysis including the correlation method are demonstrated in Sec. 6 to determine the number and symmetry of the vibrational modes for crystalline $\mathrm{NaClO}_{3}$. Also shown in Sec. 6 is an accounting method used by Fateley et ai. ${ }^{29}$ as a check on the correlation method.

\subsection{Scattering Etiiiciency, Isotropic Crystals}

The Raman scattering efficiency is defined ${ }^{31}$ to the the ratio of the number of scattered photons produced per unit time per unit cross-sectional area of the crystal in the solid angle $d \Omega$ about the direction of observation to the number of incident photons crossing a unit area in unit time. The transition probability will be proportional to the scattering efficiency. For the isotropic crystal the scattering efficiency is given by Eq. (5.1). As an illustration of the calculation of the scattering efficiency using the matrices of Table $\left[\right.$, let us consider the trigonal class $\mathrm{L}_{3 \mathrm{~d}}$. This example will be shown in detail so that it might be used as a pattern for calculating scattering efficiencies for the piezoelectric crystals of Sec. 5.3.

The irreducible representations of crystal class $D_{3 d}$ are $A_{\mathrm{lg}}$ and $E_{g}$ as shown in Table $L$. The matrices corresponding to these irreducible representations are

$A_{l g}=\left|\begin{array}{ccc}a & \cdot & \cdot \\ \cdot & a & . \\ \cdot & \cdot & b\end{array}\right| ; E_{B}=\left|\begin{array}{ccc}c & \cdot & \cdot \\ \cdot & -c & d \\ \cdot & d & .\end{array}\right| ; E_{b}=\left|\begin{array}{ccc}\cdot & -c & -d \\ -c & \cdot & \cdot \\ -d & \cdot & \cdot\end{array}\right|$.

In general, Raman spectrometer systems are designed for right angle scattering. However, it will be instructive 1, consider the scattering at some other angle, $\theta$, as shown in Fig. 15. The incident and scattered photon and the phonon wave vectors are coplanar and for convenience will define the $x z$-plane. The direction and magnitude of the phonon wave vector is specified according to Eq. (2.18). Using a gas laser as the excitation source the incident radiation will be plane polarized. The components of the scattered radiation can be measured independently by using an analyzer in the scattered beam. With the scattered radiation analyzed perpendicular to the $x z$-plane the row and column matrices describing the incident and scattered waves will be

$\hat{e}_{\mathrm{i}}=\left(e_{i}^{*}, e_{i}^{y}, o\right)$ and $\hat{e}_{y}=\left(\begin{array}{c}o \\ e_{5}^{y} \\ o\end{array}\right)$. 
Expanding Eq. (5.1), the scattering efficiency will be

$S=A\left[e_{i}^{x} R_{x y} e_{s}^{y}+e_{i}^{y} R_{y y} e_{s}^{y}\right]^{2}$.

From Eq. (5.3) one sees that the nonzero component of $R_{x y}$ is $-c$ from the second $E_{k}$ matrix. $R_{y y}$ will correspond to a from the $A_{1 g}$ matrix and - $c$ from the first $E_{g}$ matrix. If we were to restrict the incident radiation to be polarized only along the $x$-axis $\left(e_{i}^{y}=0\right)$ then $S=A[c]^{2}$ and one would be able to identify the $E_{\mathrm{s}}$ vibrations from the Raman spectrum.

With the analyzer in the scattered bear: adiusted to accept the radiation polarized parallel to the $x z$-plane the unit vectors become

$\hat{e}_{i}=\left(e_{i}^{x}, e_{i}^{y}, o\right)$ and $\hat{e}_{s}=\left(\begin{array}{c}e_{s}^{x} \\ 0 \\ e_{s}^{z}\end{array}\right)$,

and the scattering efficiency is

$S=A\left[e_{i}^{x} R_{x x} e_{s}^{x}+e_{i}^{x} R_{x i} e_{s}^{\prime}+e_{i}^{y} R_{y x} e_{s}^{x}+e_{i}^{y} R_{y z} e_{s}^{z}\right]^{2}$.

This expression can be evaluated using Eq. (5.3). If we were to make a right-angle Raman scattering measurement, $\theta=\pi / 2$ in Fig. 15, and let

$\hat{e}_{s}=\left(\begin{array}{c}0 \\ 0 \\ e_{s}^{\prime}\end{array}\right)$.

Eq. (5.7) would become

$S=A\left[e_{i}^{x} R_{x z} e_{s}^{z}+e_{i}^{y} R_{y z} e_{s}^{z}\right]^{2}=A[d-d]^{2}=0$,

assuming $e_{i}^{x}=e_{i}^{y}$. This last example illustrates, for two-or three-fold degenerate phonons, that contributions of the two or three matrices given in Table I are added to find the total scattering efficiency.

\subsection{Scattering Efficiency, Piezoelectric Crystals}

The scattering efficiency formula, Eq. (5.1), must be modified when applied to crystal vibrations that are simultaneously Raman and infrared active. The reasons for this are that in a polar crystal the longrange electrostatic forces partially lift the group-theoretical degeneracy of the lattice vibrations. More Raman lines are observed than would be predicted by the group-theoretical correlation method of Sec. 6 and illustrated in Figs. 11 and 12. The scattering efficiency is calculated on the basis of the space group of the crystal lattice corresponding to $k=0$ in Figs. 11 and 12, while the phonon frequencies observed in Raman scattering correspond to $k$ values at the right-hand edges of the figures. In addition, in unjaxial crystais some of the Raman frequencies are angular dependent. The long-range electrostatic forces also produce an electron-lattice interaction in addition to the deformation-potential interaction. This additional interaction also varies with phonon direction due to the variation in field strength. These effects require that the scattering efficiency expression be modified for polar crystals. We will consider first the case of the cubic crystal and then the uniaxial crystal.

\subsubsection{Cubic Crystals}

In cubic crystals the three-fold degenerate Raman- and infrared-active vibration will be split into a two-fold degenerate transverse and one-fold degenerate longitudinal vibration as shown in Figs. 8 to 12 . The scattering efficiency expression (5.1) can be modified to account for this mechanical polarization by a 
coordinate rotation of the axes describing the Raman tensor. For cubic crystals this simple rotation is sufficient because both the phonon displacement and electric field point in the same direction. As a result, the symmetries of the scattering produced by the two electron-lattice interactions are identical. The modified scattering efficiency for a cubic piezoelectric crystal becomes

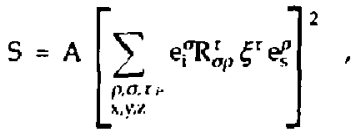

where $\xi^{\mathrm{r}}$ are scalar components of the unit vector defining the direction of the mechanical polarization of the phonon. The contributions of the matrices in Table I for a phonon of given polarization in the rotated coordinate system are summed before taking the square in $\mathrm{E}_{4}$. (5.8).

As an example of this coordinate rotation ${ }^{32}$ and the application of Eq. (5.R), consider the $F_{2}$ vibration of the $T_{d}$ cubic crystal class assuming the scattering geometry of Fig. 15. The mechanical polarization of the phonon in the direction of the phonon propagation vector (i.e., the longitudinal vibration) will have components

$\xi^{P}=(-\cos \theta / 2,0, \sin \theta / 2)$.

The transverse components will be

$\xi_{\|}=(\sin \theta / 2,0, \cos \theta / 2)$

and

$\xi_{\perp}^{\mathrm{t}}=(0,1,0)$,

for the components parallel and perpendicular to the scattering plane, respectively. For the cubic crystal it is not necessary to distinguish between $\xi$ and $\xi_{\perp}^{t}$ since these will be degenerate. The incident wave has components $\hat{e}_{i}=\left(e_{i}^{x}, e_{i}^{y}, o\right)$ and the scattering wave will have components $e_{s}^{l}=(\cos \theta, 0,-\sin \theta)$ and $e_{s}{ }_{s}^{1}=(0,1,0)$ parallel and perpendicular to the $x z$-plane, respectively.

For purposes of illustration, the scattering efficiency calculation will be shown in detail for the case of longitudinal phonon excitation and the scattered light analyzed parallel to the $x z$-plane.

$S^{f}=A\left[\left(e_{i}^{x}, e_{i}^{y}, o\right)\left\{F_{2}(x)(-\cos \theta / 2)+F_{2}(y) \cdot 0+F_{2}(z) \sin \theta / 2\right\}\left(\begin{array}{c}\cos \theta \\ 0 \\ \sin \theta\end{array}\right)\right]^{2}$.

Here $F_{2}(\sigma)$ signifies the matrices corresponding to the irreducible representation of the same symbol. The product $F_{2}(x)(-\cos \theta / 2)$ is a rotation by an angle of $\theta / 2$ about the $y$-axis corresponding to the longitudinal phonon vibration. Substituting the matrices of Table I into Eq. (5.12) and performing the multiplications we have

$S_{1}^{\ell}=A^{\prime} d^{2}\left(e_{i}^{y}\right)^{2}(\sin 3 \theta / 2)^{2}$.

For incident radiation plane polarized along the $x$-axis, $e_{i}^{y}=0$ and $S_{\|}^{p}=0$, so that the $F_{2}$ vibration would not be excited. The remaining scattering efficiencies are calculated in a manner similar to that for $S_{\|}^{p}$ with the following results

$S_{\perp}^{\ell}=A^{\prime}\left[e_{i}^{x} d \sin \theta / 2\right]^{2}$.

$\left.S_{1}^{t}=\operatorname{Ad}^{2} \mid\left(e_{i}^{y} \cos 3 \theta / 2\right)^{2}+\left(e_{1}^{x} \sin \theta\right)^{2}\right]$,

and

$S_{-}^{t}=\operatorname{Ad}^{2}\left[\mathbf{e}_{i}^{\mathrm{x}} \cos \theta / 2\right]^{2}$. 
The constant of proportionality for longitudinal vibrations in Eqs. (5.13) and (5.14) is different from that for the transverse vibrations in Eqs. (5.15) and (5.16) because the electric field associated with the polar lattice vibration provides an additional electron-lattice coupling term. The transverse phonon is unaffected by this electric field as shown in Fig. 8.

\subsubsection{Uniaxial Crystals}

For uniaxial crystals the lattice displacement which determines the deformation potential scattering will not, in general, be parallel to the electrostatic field which controls the polar scattering. As a result the scattering formula of Eq. (5.8) is further modified ${ }^{32}$ by including a second matrix rotation and the result is

$S=\left[\sum_{\sigma, \rho, t} \mathrm{e}_{\mathrm{i}}^{\sigma} \mathrm{R}_{\sigma \rho}^{\tau}\left(\alpha \xi^{r}+\beta \mathrm{k}^{2}\right) \mathrm{e}_{\mathrm{s}}^{\ell}\right]^{2}$,

where $\alpha$ and $\beta$ are coefficients proportional to the deformation potential and electric field strength, respectively. $\dot{\xi}$ is a unit vector in the direction of the lattice displacement and $\bar{k}$ is a unit vector parallel to the electric field. In general, $\bar{\xi}$ is not parallel to $\bar{k}$ in a unjaxial crystal. For a cubic crystal $\bar{\xi}$ and $\hat{k}$ are parallel for longitudinal phonons and $(\alpha+\beta)^{2}=A^{\prime}$ of Eqs. (5.13) and (5.14). The electrostatic field does not couple with the transverse phonons as shown in Sec. 2 and as a result $\beta=0$ for both cubic and uniaxial crystals for the transverse phonons.

As an example of the application of the scattering formula (5.19) let us consider first the case of a crystal with the electrostatic force predominating the anisotropic force as shown in Fig. 11. For this case the electric field vector will be approximately collinear with the lattice displacement, $\hat{\xi} \sim \hat{\mathrm{k}}$. The components of $\bar{\xi}$ will be the same as Eqs. (5.9)-(5.11) with the exception that for the uniaxial crystals Eq. (5.10) will conespond to the extraordinary wave and $\mathrm{Eq},(5.11)$ the ordinary wave. The photon and phonon waves in a uniaxial crystal will be only approximately longitudinal or transverse because of the birefringence. Assuming the geometry of Fig. 15, the scattering efficiency for a semi-transverse scattered wave and a semi-longitudinal phonon in a crystal of $\mathrm{C}_{4 \mathrm{v}}$ symmetry will be

$S=(\alpha+\beta)^{2}\left[\left(e_{i}^{x}, e_{i}^{y}, 0\right)\left[\xi_{x}^{P} E(x)+\xi_{y}^{P} E(y)+\xi_{x}^{R} A(z)\right]\left(\begin{array}{c}\cos \theta \\ 0 \\ -\sin \theta\end{array}\right)\right]^{2}$,

where $E(x), E(y)$, and $A(z)$ represent the matrices corresponding to the irreducible representations of Table I. Making the substitutions from Table I, the scattering efficiency becomes

$S^{f}=(\alpha+\beta)^{2}|a \sin \theta / 2 \cos \theta+e \cos \theta / 2 \sin \theta|^{2}\left(e_{i}^{x}\right)^{2}$.

indicating that both the $A$ and $E$ modes will be excited. For scattered light perpendicular to the $x z$-plane the scattering efficiency will be

$S_{-}^{f}=(\alpha+\beta)^{2}\left(e_{i}^{y} a \sin \theta / 2\right)^{2}$.

showing that only the $A$ modes are excited. The semi-transverse extraordinary phonon has a negligible electric field so that $\beta=0$ in Eq. (5.17) and the scattering efficiencies are

$S^{t}=\left[a e_{i}^{x} \cos \theta / 2 \cos \theta-e e_{i}^{x} \sin \theta / 2 \sin \theta\right]^{2} \alpha^{2}$

and

$S_{\perp}^{i}=a^{2}\left[e_{i}^{y} a \cos \theta / 2\right]^{2}$. 
The scattering efficiencies for the transverse ordinary phonons $(\beta=0)$ become

$S_{i}^{t}=\left|e_{i}^{y} e \sin \theta\right|^{2} \alpha^{2}$.

and

$S_{1}^{t}=0$

illustrating in the one case that only E modes are excited and in the second case that no Raman-active modes will be excited.

Let us now consider the case of Fig. 12 corresponding to the polar uniaxial crystal with the anisotropy of the short-range forces predominating over the electrostatic forces. For this case the two extraordinary phonons have lattice displacements either approximately parallel or perpendicular to the optic axis of the crystal. The electrostatic field will be parallel to the phonon propagation direction. For the extraordinary phonon corresponding to a lattice displacement parallel to the c-axis we have

$\bar{\xi}=(0,0,1)$.

The coefficient $\beta$ is proportional to the magnitude of the electric field along the coordinate directions so that the $z$ component will be $\beta \sin \theta / 2$ and

$\hat{k}=(-\cos \theta / 2,0, \sin \theta / 2)$.

The scattering efficiency of Eq. (5.17) becomes

$\mathrm{S}=\left[\sum_{\tau, \rho, \mathrm{r}} \mathrm{e}_{\mathrm{i}}^{\sigma} \mathrm{R}_{\sigma \rho}^{\mathrm{r}}\left(\alpha \xi^{\tau}+\beta \sin \theta / 2 \mathrm{k}^{\tau}\right) \mathrm{e}_{\mathrm{s}}^{\rho}\right]^{2}$.

The parallel and perpendicular scattering efficiencies become

$S=\left\{\mathbf{e}_{1}^{\mathrm{x}} \mathrm{a}\left[\alpha+\beta \sin ^{2} \theta / 2\right] \cos \theta+\mathbf{e}_{i}^{\mathrm{x}} \mathrm{e} \beta \sin \theta / 2 \cos \theta / 2 \sin \theta\right\}^{2}$

and

$\mathrm{S}_{\perp}=\left\{\mathrm{e}_{\mathrm{i}}^{\mathrm{y}} \mathrm{a}\left|\alpha+\beta \sin ^{2} \theta / 2\right|\right\}^{2}$.

For the extraordinary phonon with displacement perpendicular to the c-axis and along the $x$-axis

$\bar{\xi}=(1,0,0)$

and

$\beta \rightarrow-\beta \cos \theta^{\prime} 2$.

Th. scattering coefficients are

$S=\left\{e_{1}^{x} a \beta \cos \theta / 2 \sin \theta / 2 \cos \theta-e_{i}^{x} e\left(\alpha-\beta \cos ^{2} \theta / 2\right) \sin \theta\right\}^{2}$

and

$S_{.}=\left\{\mathrm{e}_{1}^{\mathrm{x}} a \beta \cos \theta / 2 \sin \theta / 2\right\}^{2}$. 
The scattering ufficiencies for the ordinary phonon are the same as for the case with the predominate electrostatic forces and are given by Eqs. (5.22) and (5.23).

\subsection{Scattering Efficiency, $\mathrm{NaClO}_{3}$}

The value of the scattering efficiency lies in the ease of identifying the particular vibrational species in the Raman spectrum. The $\mathrm{C}_{4 \mathrm{v}}$ uniaxial crystal of Sec. 5.3.2 is an illustration of this property. Given in this section are the scattering efficiencies for crystalline $\mathrm{NaClO}_{3}$ and we will illustrate how the various vitrational species can be identified from the Raman spectrum. ${ }^{3.1}$

Crystalline sodium chlorate, $\mathrm{NaClO}_{3}$, belongs to the cubic space group $\mathrm{T}^{4}(\mathrm{P} 2,3)$ with 4 molecules per primitive cell. ${ }^{34}$ From Table $I$ it is seen that the $\mathrm{F}$ vibrations are simultaneously Raman and infrared active while the $\mathrm{A}$ - and $\mathrm{E}$-species vibrations are only Raman active. Therefore, the scattering efficiency is given by Eq. (5.8) for the F phonons and by Eq. (5.1) for the A and E phonons. The Raman tensors for crystal symmetry class $T$ of Table I are repeated here to be

$$
\begin{aligned}
& A=\left|\begin{array}{ccc}
a & \cdot & \cdot \\
\cdot & a & \cdot \\
\cdot & \cdot & b
\end{array}\right|: \mathbf{E}=\left|\begin{array}{ccc}
b & \cdot & \cdot \\
\cdot & \mathbf{b} & \cdot \\
\cdot & \cdot & -2 b
\end{array}\right|+\left|\begin{array}{ccc}
-b \sqrt{3} & \cdot \\
\cdot & -b \sqrt{3} & \cdot \\
\cdot & \cdot & \cdot
\end{array}\right| \\
& F(x)=\left|\begin{array}{ccc}
\cdot & \cdot & . \\
\cdot & \cdot & d \\
. & d & .
\end{array}\right| ; F(y)=\left|\begin{array}{ccc}
\cdot & \cdot & d \\
\cdot & \cdot & . \\
d & \cdot & .
\end{array}\right| ; F(z)=\left|\begin{array}{ccc}
\cdot & d & . \\
d & \cdot & . \\
\cdot & . & .
\end{array}\right| \text {. }
\end{aligned}
$$

For right-angle Raman scattering the notation describing the incident and scattered polarizations is as follows. ${ }^{19}$ For example, $y(x z) x$ indicates a measurement of the Raman tensor element $R_{x a}$ scattering along the $x$-crystallographic axis with light incident in the $y$ direction. The subscripts of the $R_{x z}$-tensor element indicate that the incident light is plane polarized parallel to the $x$ axis and the scattered light is plane polarized parallel to the $z$ axis.

Using Eqs. (5.1) and (5.8) and the Raman tensors of Eq. (5.29), we can show that the spectrum z $(y x) y$ will contain only the $F(z)$ mode corresponding to transversely polarized phonons. The polarization vectors of the incident and scatlered light are $\hat{\mathbf{c}}_{\mathrm{i}}=(0,1,0)$ and $\hat{\mathbf{e}}_{\mathrm{s}}=(1,0,0)$, respectively. The scattering geometry is shown in Fig. 16. Using Eq. (5.1) and the $A$ and $E$ matrices of Table $I$ the scattering efficiency will be

$S=A\left|e_{i}^{y} R_{y x} e_{,}^{x}\right|^{2}=A(1 \cdot 0 \cdot 1)=0$,

since the $R_{y x}$ element of the matrix $A+E$ is zero. For the F matrices, Eq. (5.8) is the scattering formula since these vibrations are polar. From Eq. (5.8) the scattering is

$S=A\left[e_{i}^{y}\left(R_{y x}^{x} \xi^{x}+R_{y x}^{y} \xi^{y}+R_{y x}^{z} \xi^{x}\right) e_{s}^{x}\right]^{2}$

where

$\xi^{p}=\left(0,-\frac{1}{\sqrt{2}}, \frac{1}{\sqrt{2}}\right) ; \xi_{\|}^{\prime}=\left(0,-\frac{1}{\sqrt{2}},-\frac{1}{\sqrt{2}}\right)$ and $\xi_{\perp}^{\prime}=(1,0,0)$.

For the longitudinally polarized phonon, $\xi^{\rho}$, Eq. (5.30) becomes

$S^{E}=A\left[e_{i}^{y}\left(R_{y, x}^{x} \xi_{x}^{i}+R_{y x}^{y} \xi_{y}^{P}+R_{y x}^{\prime} \xi_{y}^{P}\right) \mathbf{e}_{y}^{x}\right]^{2}=\frac{A}{2} d^{2}$.

The first term vanishes berause $\xi_{x}^{r}=0$, the second term is zero because $R_{y x}^{y}=0$ in $f(y)$ and the remaining term is nomzero. This shows that only the $f(x)$ vibration will he excited. 
Similar calculations will show that the spectra $z(x z) y$ and $z(y z) y$ will contain $F(y)$ and $F(x)$ modes, respectively, corresponding to both longitudinal and transverse phonons.

The $A$ and $E$ modes are not infrared active so that the scattering e'fficiency will be given by Eq. (5.1). Let us take $\hat{e}_{i}=(1,1,0)$ and $\hat{\mathbf{c}}_{\mathrm{s}}=(1, \bar{l}, 0)$, then only the $\mathrm{E}$ vibrations will have a nonzero scattering efficiency and the $E$ species can be identified from the $z\left(y^{\prime} x^{\prime}\right) y^{\prime}$ spectrum where $x^{\prime}$ and $y^{\prime}$ are axes $45^{\circ}$ relative to the $x$ and $y$ axes and rotated about the $z$ axis. Spectra measured for $\hat{e}_{i}=(1,0,1)$ and $\hat{\mathrm{e}}_{i}=(1,0, \overline{\mathrm{l}})$, and $\hat{\mathrm{e}}_{1}=(0,1,1)$ and $\hat{e}_{4}=(0, \overline{\mathrm{l}}, 1)$ will also yield only E-species vibrations. For $\mathrm{NaClO}_{3}$ one cannot excite only the $A$ vibration. The spectra $z(x x) y, z(y y) x$, and $x(2 z) y$, for example, will contain $A+E$ and by comparison of these spectra with the $z\left(y^{\prime} x^{\prime}\right) y^{\prime}$ spectrum (E species) the $A$ vibrations can be identified.

\section{Prediction of Raman Spectrum of $\mathrm{NaC}_{3} \mathrm{O}_{3}$}

The number and symmetry species of the Raman- and infrared-active modes in jonic crystals can be predicted using the factor group analysis and correlation method as outline in Sec. 5.1. This determination will be demonstrated for the case of sodium chlorate.

As stated in $\mathrm{Sec}$. 5.4, $\mathrm{NaClO}_{3}$ belongs to the cubic space group $\mathrm{N}^{\circ} 198, \mathrm{~T}^{4},(\mathrm{P} 2,3)$ with 4 molecules per primative cell. The site symmetries ${ }^{34}$ of the sodium and chlorate ions are both $C_{3}$. The point symmetry of the free chlorate ion ${ }^{35}$ is $\mathrm{C}_{3 v}$ and the factor group symmetry is $\mathrm{T}$ corresponding to the space group $\mathrm{T}^{4}$. The $\mathrm{NaClO}_{3}$ molecule will be decomposed into a monatomic ion $\left(\mathrm{Na}^{+}\right)$and a polyatomic ion $\left(\mathrm{ClO}_{3}^{-}\right)$for the analysis that follows.

\subsection{Chlorate Ion, Internal Vibrations}

The free chlorate ion of $C_{3 v}$ symmetry is to be correlated with the species of the sile group symmetry $C_{3}$ and the factor group symmetry $T$. From the character table corresponding to $C_{3 v}$ symmetry, Table II, it can be seen that the translatory modes belong to species $A_{1}$ and $E$ and the rotatory modes belong to species $A_{2}$ and $E$. The correlation $C_{3 v} \rightarrow C_{3} \rightarrow T$ is shown in Table III. The published correlation tables of Fateley et al. ${ }^{29}$ were used for this step but the character tables ${ }^{30}$ of $C_{3 y}, C_{3}$, and $T$ could have also been used. The free chlorate ion has six (3n-6 = 3 $\times 4-6=6$ ) internal modes of vibration consisting of four frequencies of two different symmetry species. ${ }^{3.5}$ These internal modes are labeled $v_{1}\left(A_{1}\right), v_{2}\left(A_{1}\right), v_{3}(E)$, and $v_{4}(\mathrm{E})$ and are shown in Table III. The rotatory and translatory external frequencies are also shown in the table. From the last column of Table $l i l$ it is seen that the frequencies and symmetries of the internal modes of the chlorate ion in the crystal will be

$$
\Gamma_{\text {iniernal }}=2 \mathrm{~A}\left(v_{1}, v_{2}\right)+2 \mathrm{E}\left(v_{3}, v_{4}\right)+6 \mathrm{~F}\left(v_{\mathrm{t}}, v_{2}, 2 v_{3}, 2 v_{4}\right)
$$

\subsection{Sodium Ion Vibrations}

The symmetry species of the sodium ions could be determined using the procedure of Sec. 6.1 but it will be instructive to use the method of Fateley et al. ${ }^{29}$ Identical results are obtained by either method but the bookkeeping is easier by the Fateley method.

The site symmetry of the sodium ion is $C_{3}$ and this is to be correlated with the factor group symmetry $T$ of the crystal. This correlation is shown in Table IV. The columns headed $f^{\gamma}, t^{\gamma}, C_{\zeta}, a_{\zeta}, a_{A}+a_{E}$ are used in checking the correlation method. The number of translations corresponding to symmetry species $\gamma$ is indicated by $t^{\gamma}$. From the $C_{3}$ character table, ${ }^{29,30}$ a translation in the $z$ direction ( $T$, ) will correspond to the A species; thus the 1 under $t^{\gamma}$ in Table IV. The degrees of freedom for species $\gamma$ are given in the first column labeled $\mathrm{f}^{\prime}\left(=\mathbf{n t}^{r}\right)$, which is the number of translations times the number of ions per unit cell. For the $A$ species we have $f^{y}=4 \times 1=4$. From the $C_{3}$ character table the $E$ species corresponds to two 
Table III. Correlation diagram for the chlorale modes of sodium chlorate.

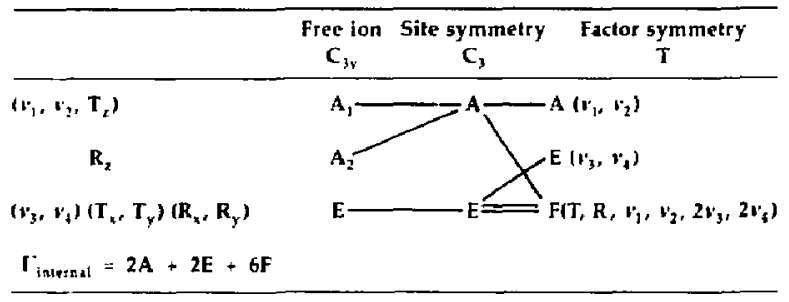

Table IV. Correlation diagram for the sodium ion modes of sodium chlorate.

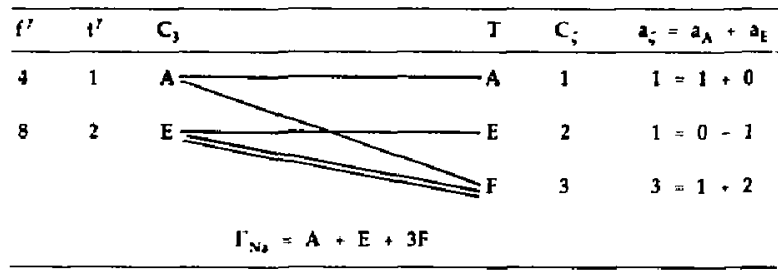

translations ( $T_{x}$ and $T_{y}$ ) so $t^{\gamma}=2$ and $f^{\gamma}=4 \times 2=8$. As the first check, the sum of the degrees of freedom for each species must equal the total degrees of freedom for all the ions in the unit cell (4 $\mathrm{Na}$ ions $\times 3$ degrees per atom $=12$ total degrees $=4$ (A species $)+8(E$ species $)=12)$. The column labeled $C_{5}$ gives the degeneracy of the species $\zeta$. The usual convention is used $(A, B \rightarrow 1, E \rightarrow 2, F \rightarrow 3$, etc.). The columns labeled $a_{A}$ and $a_{E}$ indicate the number of lines originating from species $A$ and $E$ and terminating on each species under $T$. In the first case, $i$ is seen that $a_{A}=1$ and $a_{E}=0$ with the sum given by $a_{5}$. In group theory language, $a_{y}=a_{A}+A_{E}$ are the coefficients relating the irreducible representation to the reducible representation. Another way of expressing it is that $a_{\xi}$ is the number of lattice vibrations of the equivalent set of atoms or ions in species $\zeta$ of the factor group. The total number of vibrations of the equivalent sodium ions will therefore be

$\Gamma_{\text {oddium }}=\sum_{\zeta} a_{\Xi} \zeta \zeta=1 \mathrm{~A}+1 \mathrm{E}+3 F$.

As a check, the sum

$\sum_{\underline{b}} \mathbf{a}_{\zeta} c_{\zeta}$

must equal the total degrees of freedom of the sodium ion. From Table IV this sum is $1 \times 1+1 \times 2+3 \times 3$ $=12=3 \times 4$. This example jllustrates the value of the intermal checking capability of the Fateley method. Thi sane prosedure will now low used for determining the external vibrations of the chlorate ion. 


\subsection{Chlorate Ion External Vibrations}

The site symmetry of the chlorate ions are $C_{1}$, the same as the sodium ions. 'This means that the correlation table for the chlorate ion will be the same as that given in Table IV and the chlorate in external vibrations will be of the same form as Eq. (6.2). We have for the chlorate ions

$\Gamma_{C O_{1}}=A+E+3 F$.

\subsection{Rotatory Spectrum}

The frequency spectrum arising from the rotatory motions of the sodium and chlorate ions can easily he predicted by using Table IV. From the character table for point group $C_{3}$ it is seen that species $A$ corresponds to the $R_{z}$ and species $E$ corresponds to rotations $R_{x}$ and $R_{y}$. The character table for the rotatory motion is identical to Table IV but with $\mathrm{t}^{\gamma}$ replaced by $\mathrm{R}^{\gamma}$ indicating the rotational motion corresponding to species $\gamma$. $f^{\gamma}$ will now be the total rotational degrees of freedom for each species. For the example illustrated here

$\sum_{\gamma} f^{r}=12$

Because of the similarity of the correlation tables for the translatory and rotatory motions we will use Table IV. The rotatory spectrum for the sodium and chlorate ions will be

$\Gamma_{\mathrm{rot}}=\mathrm{A}+\mathrm{E}+3 \mathrm{~F}$.

The internal check on the accuracy of Eq, (6.4) is the same as that given in Sec. 6.2.

\subsection{Acoustical Vibrations}

From the character table of the point group $T$ the three pure translations of the lattice belong to the species $F$. For the Raman spectrum we are interested only in the optical vibrations and the pure translations correspond to acoustic modes. We therefore substract the acoustic modes from the translational modes.

\subsection{The Spectrum for $\mathrm{NaClO}_{3}$}

The total vibrational spectrum is given by Eqs. (6.1), (6.2), (6.3), (6.4) and the acoustic modes; the results are summarized in Table V. The lattice vibrations are the sum of Eqs. (6.2) and (6.3). The infrared and Raman activities, indicated in the last column of the table, are deternined by consulting the transformation properties of the translational unit vectors and their direct products as listed in the character table for point group $T$. From the character table it is seen that only the $F$ species will be infrared active while the $A, E$, and $F$ species will be Raman active. It should be noted that only the $F$ species are simultaneously infrared and Raman active. The results of the factor group analysis indicates that there should be at least 24 Raman lines in the spectrum. The analysis does not predict the additional number of lines to be expected because of degeneracy removed by the electrostatic forces for the $F$ species. The use of several scattering geometries can sometimes assist in identifying the TO and LO modes as shown in Sec. 5.4.

Shown in Fig. 17 is the Raman spectrum of $\mathrm{NaClO}_{3}$ taken by Hartwig et al. ${ }^{25}$ Similar spectra have been meastred by She et al. ${ }^{36}$ and Nicola et al. ${ }^{37}$ The scattering geometry of Fig. 17(a) is $z\left(y^{\prime} x^{\prime}\right) y^{\prime}$ and the 
Table V. Number and symmetry of the Raman- and infraredactive vibrations for crystalline $\mathrm{NaClO}_{3}$.

\begin{tabular}{|c|c|c|c|c|c|}
\hline $\begin{array}{c}\text { Crystal } \\
\text { symmetry }\end{array}$ & $\begin{array}{c}\text { Lattice } \\
\text { vibrations }\end{array}$ & $\begin{array}{l}\text { Acoustical } \\
\text { vibrations }\end{array}$ & Rotations & $\begin{array}{l}\text { Intramolecular } \\
\text { vibrations }\end{array}$ & $\begin{array}{l}\text { Spectral } \\
\text { activity }\end{array}$ \\
\hline $\mathrm{T}$ & & & & & 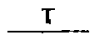 \\
\hline A & 2 & & 1 & 2 & $\mathbf{R}$ \\
\hline E & 2 & & t & 2 & $\mathbf{R}$ \\
\hline $\mathbf{F}$ & 6 & 1 & 3 & 6 & $\mathbf{R}$, IR \\
\hline
\end{tabular}

Table VI. Raman-shifted frequencies in crystalline $\mathrm{NaClO}_{3}$ al $294^{\circ} \mathrm{K}$.

\begin{tabular}{|c|c|c|c|}
\hline $\begin{array}{c}\text { Frequency } \\
\text { shift } \\
\text { (cm ') }\end{array}$ & \multicolumn{2}{|c|}{ Symmetry } & Species \\
\hline 65 & $\mathbf{E}$ & $\mathrm{m}^{\mathbf{n}}$ & \\
\hline 73 & $F(T O)$ & $\mathbf{m}$ & \\
\hline 77 & F (LO) & $\mathbf{m}$ & \\
\hline 84 & $\mathbf{A}$ & $\mathbf{m}$ & \\
\hline 93 & $\mathbf{F}$ & $\mathrm{m}$ & Lattice modes \\
\hline 120 & $\mathbf{F}$ & s & $2 A+2 E+5 F$ \\
\hline 126 & $\mathbf{F}$ & $\mathbf{s}$ & \\
\hline 128 & $\mathbf{F}$ & $\mathbf{s}$ & \\
\hline 132 & A & $\mathrm{m}$ & \\
\hline 177 & $\mathbf{E}$ & m & \\
\hline 192 & $\mathbf{F}$ & $w$ & Rotation \\
\hline 482 & $\mathbf{E}$ & $\mathbf{s}$ & Intramolecular \\
\hline 488 & $\mathbf{F}$ & 5 & $E+2 F$ \\
\hline 619 & A & $s$ & \\
\hline 624 & $F(T O)$ & $\mathbf{s}$ & Intramolecular \\
\hline 629 & F (LO) & $\mathbf{s}$ & $A+F$ \\
\hline 720 & $\mathbf{F}$ & $w$ & \\
\hline 919 & $A$ & $w$ & Rotalion \\
\hline 932 & $\mathbf{A}$ & $\mathbf{s}$ & \\
\hline 937 & $A+F(T O)$ & $s, w$ & Intramolecular \\
\hline 940 & $F(\mathbf{L O})$ & $w$ & $A+\mathbf{F}$ \\
\hline 957 & $\mathbf{E}$ & $\mathbf{m}$ & \\
\hline 966 & $\mathbf{F}$ & $\mathbf{m}$ & Intramolecular \\
\hline 986 & $F(T O)$ & $\mathbf{m}$ & $E+2 F$ \\
\hline 1025 & $F(L O)$ & $\mathbf{m}$ & \\
\hline 1062 & E & $\mathbf{w}$ & Rolation \\
\hline 1085 & F & $w$ & Fotanon \\
\hline
\end{tabular}

- Approximate relative intensities: weak (w), medium (m), strong (s). 
analysis of Sec, 5.4 shows that the resulting spectrum will correspond to E vibrations. The geometry of Fig. 17(b) is $z(x x) y$ and corresponds to $A+E$ vibrations. Comparing (a) with (b) one can identify the $A$ modes. The scattering geometries of (c) and (d) will ixcite F (TO) and F (LO) modes, respectively. By this procedure each of the Raman lines has been identified and the results ${ }^{36}$ are summarized in Table Vl. 


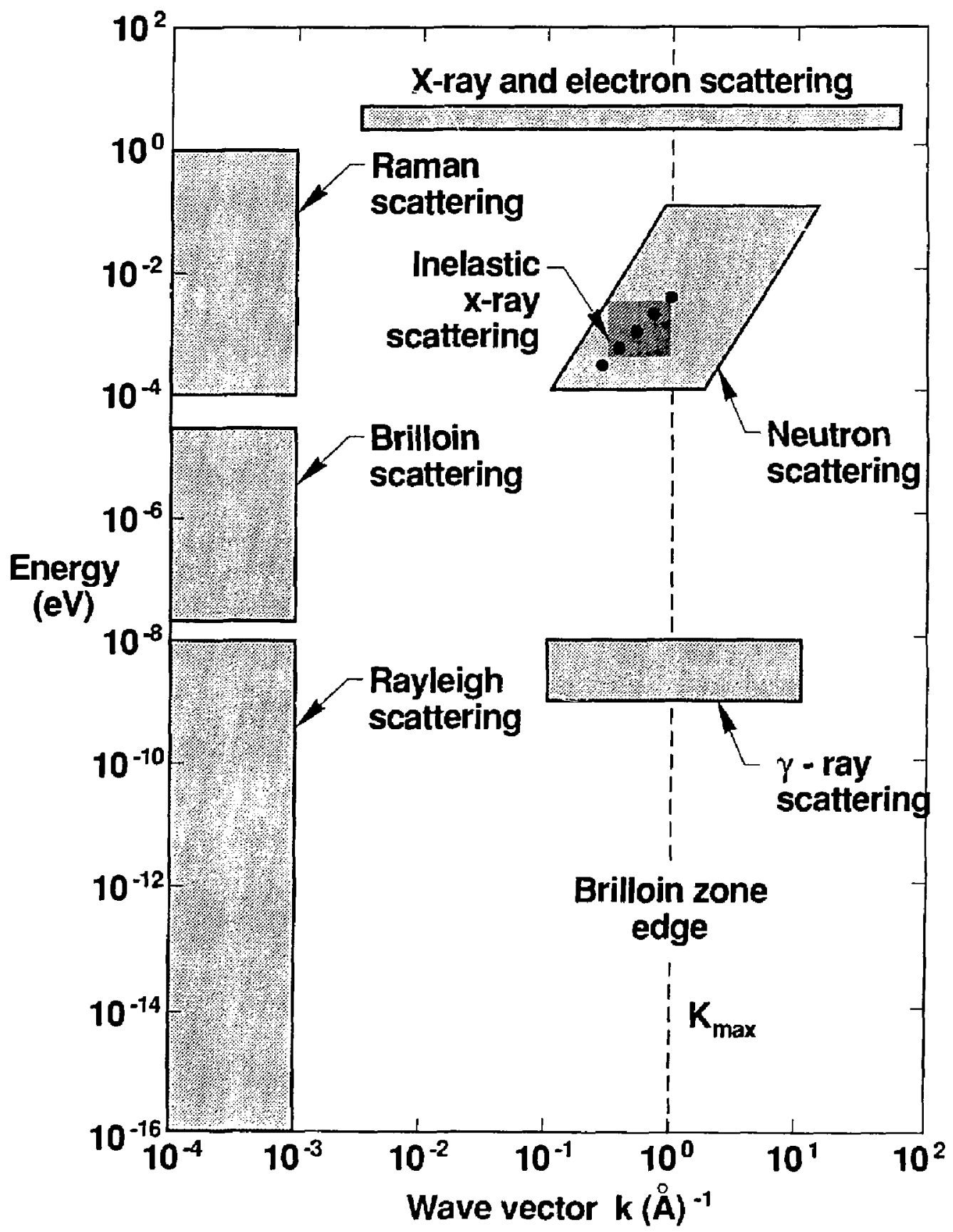

Figure 1. Frequency-wave vector diagram showing areas of applicability of different processes. 


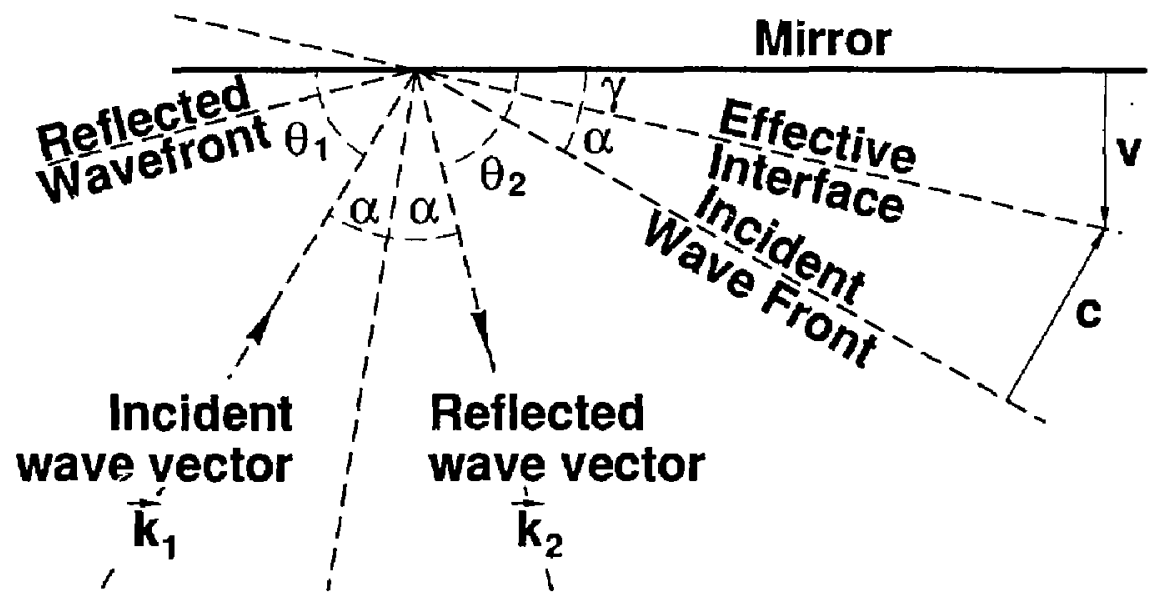

Figure 2. Reflected plane wave from a moving mirror.

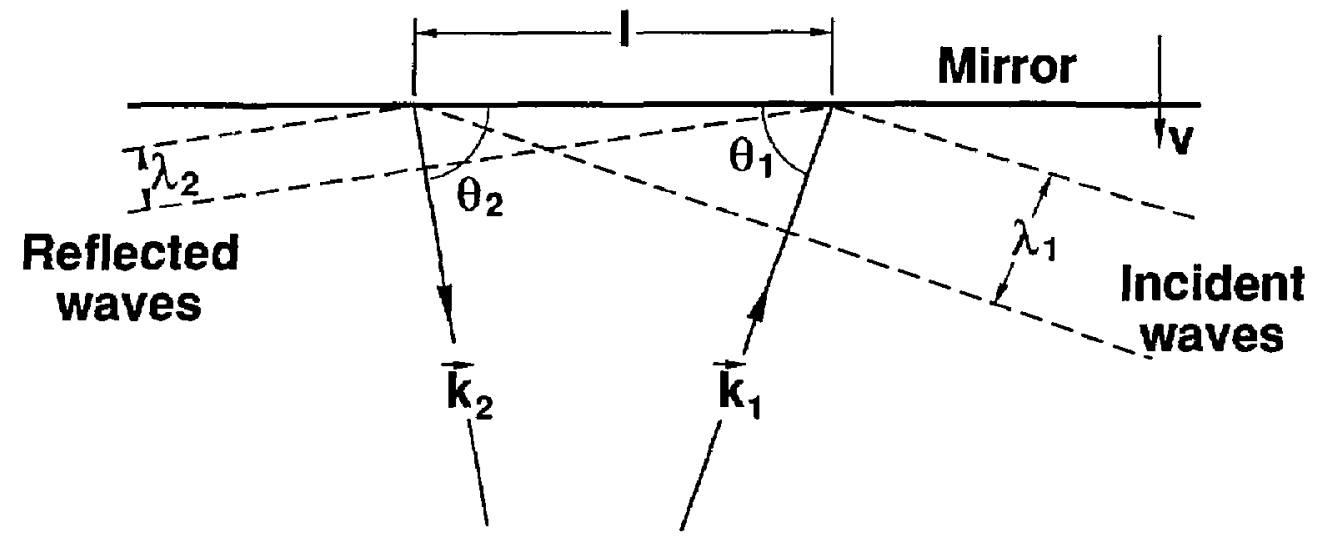

Figure 3. Doppler shift of wave reflected from a moving mirror. 


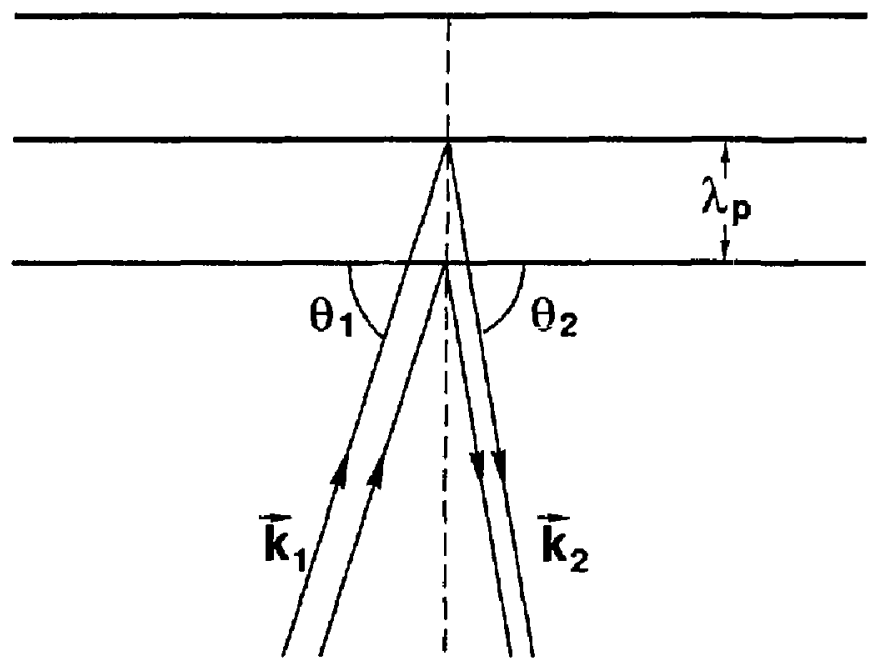

Figure 4. Interference from waves reflected from adjacent mirrors.

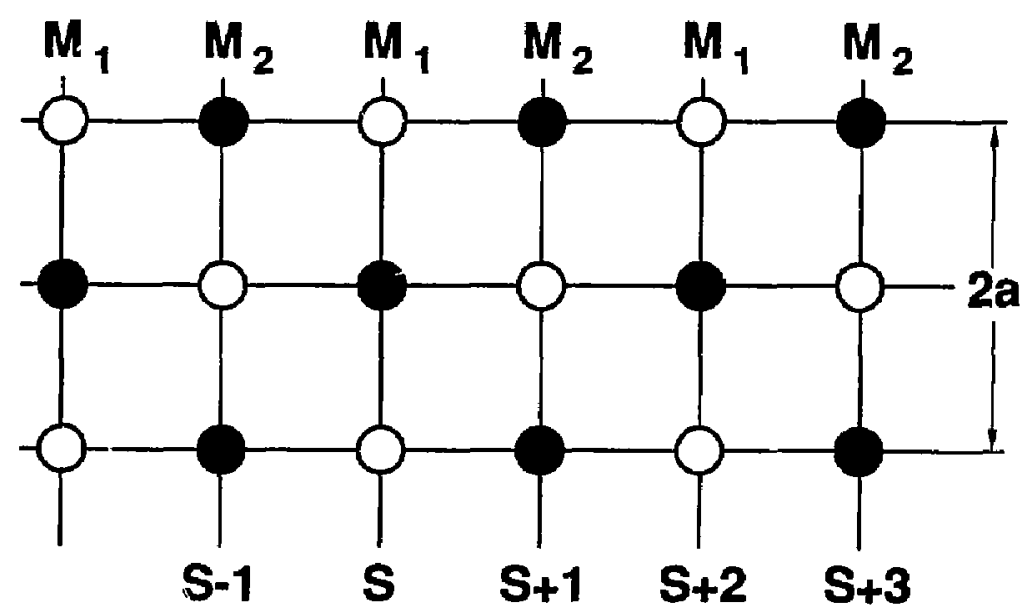

Figure 5. Cubic diatomic linear molecule. 


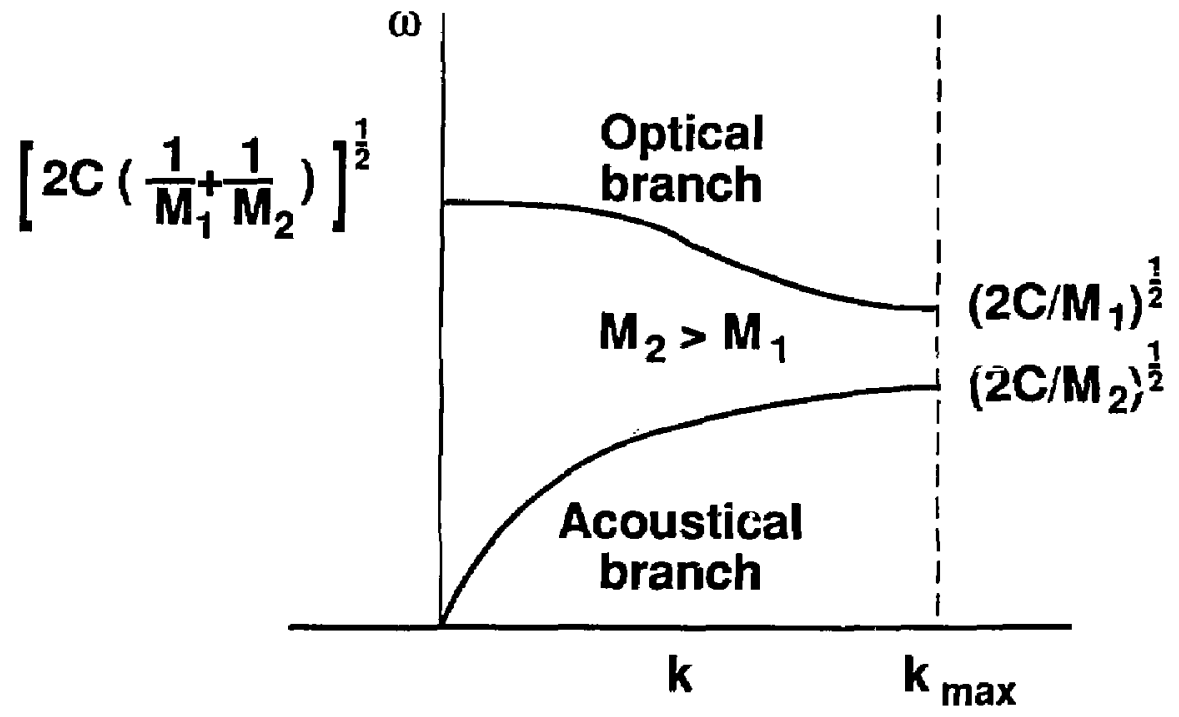

Figure 6. Dispersion relation for the diatomic molecule.

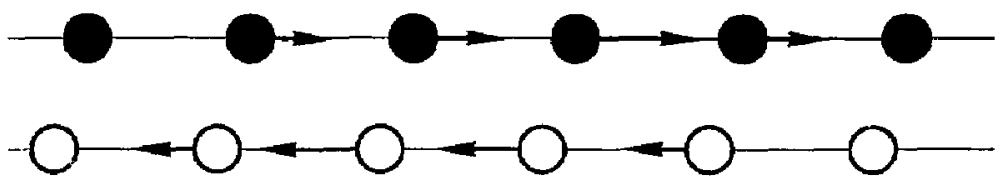

Longitudinal mode
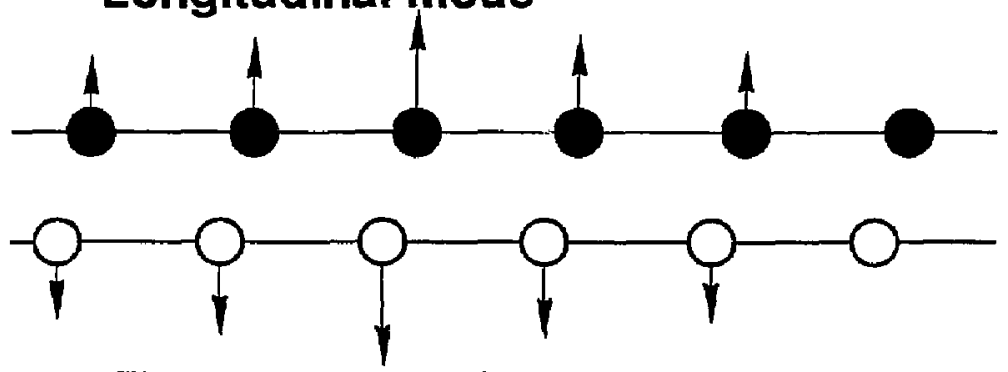

Transverse mode

\section{Anion \\ Cation}

Figure 7. Longitudinal and transverse optical modes of the diatomic linear molecule. 


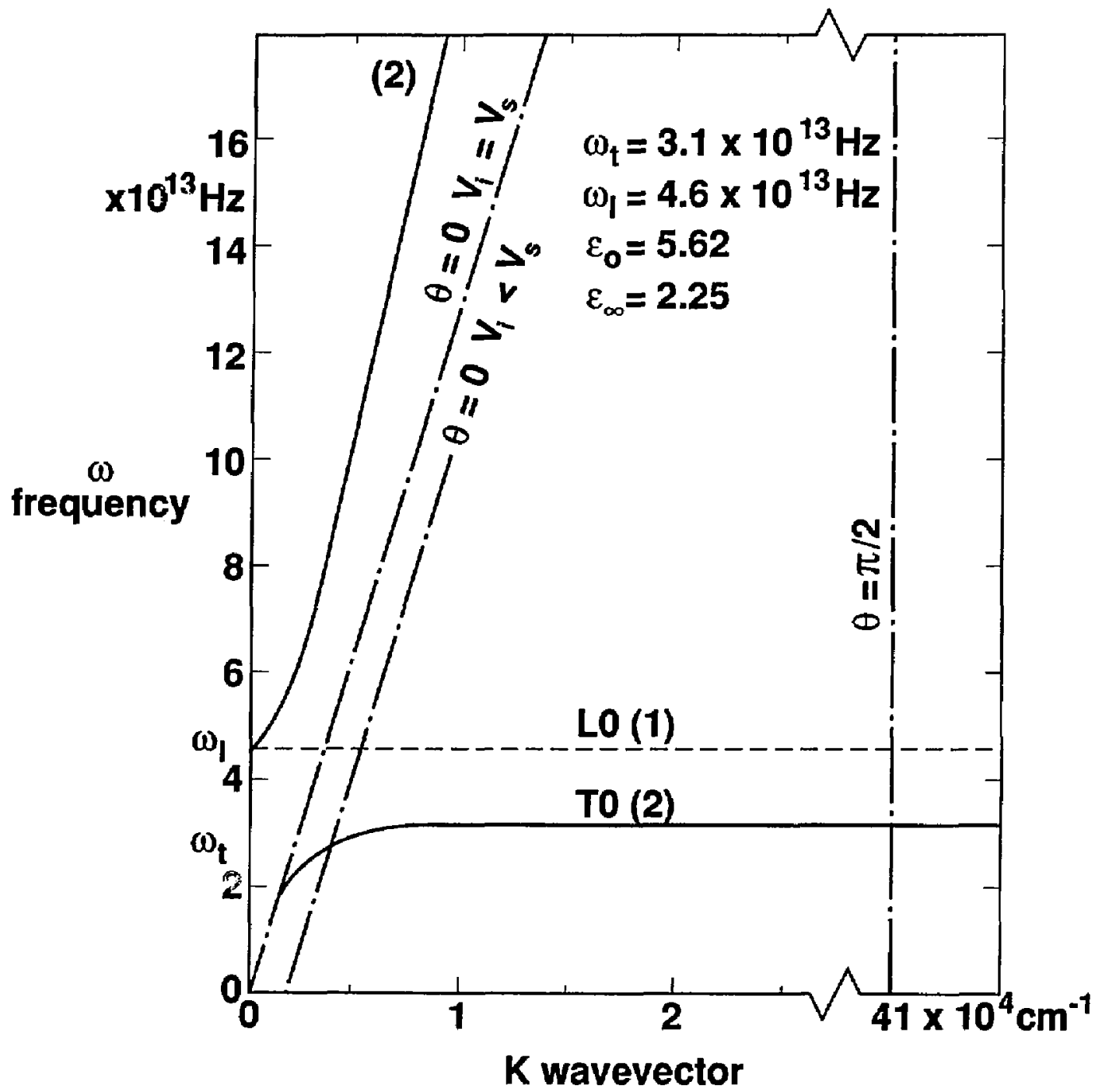

Figure 8. Long wavelength region of the optical branch with independent phonon and photon waves. 


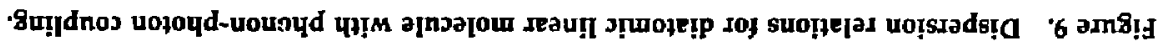
L-US Y

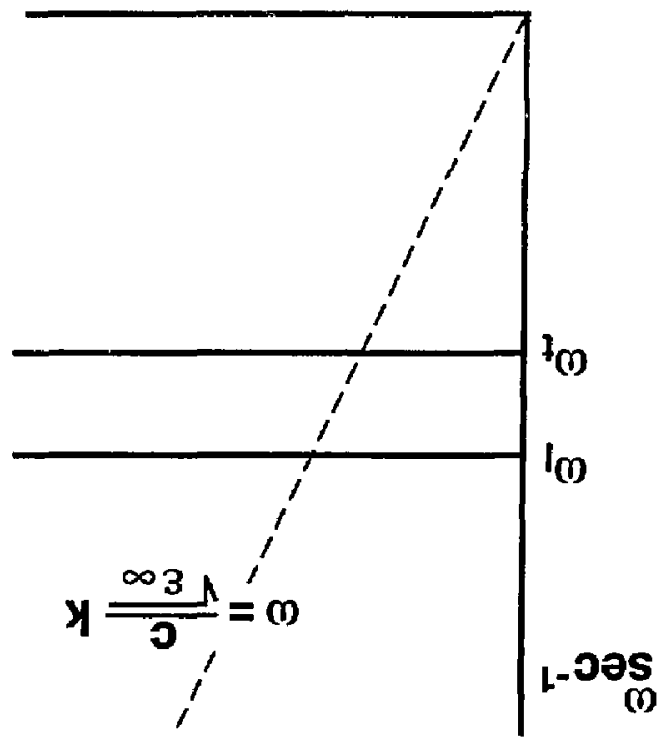




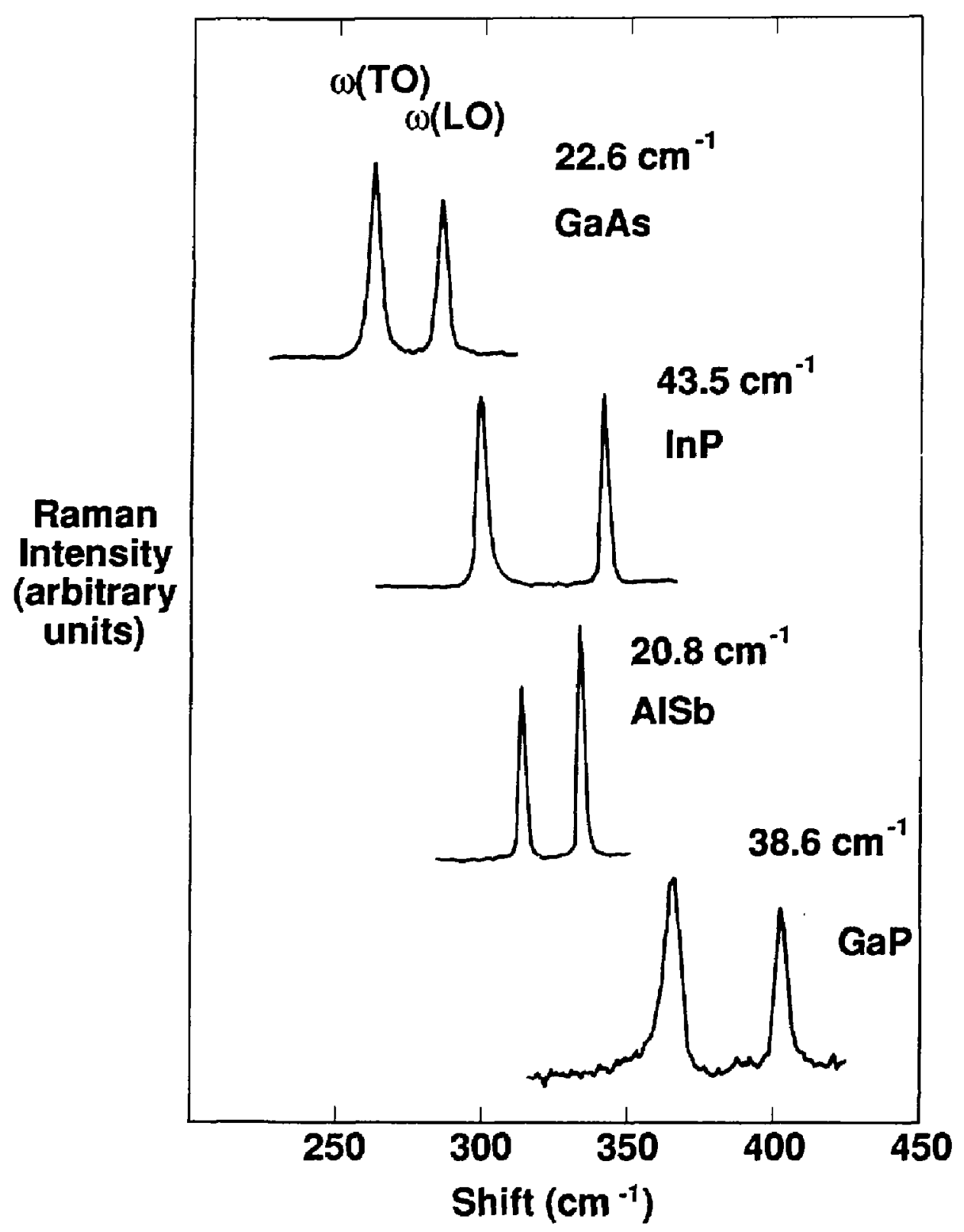

Figure 10. Raman spectra of GaAs, InP, AlSb, and GaP from Ref. I7. 

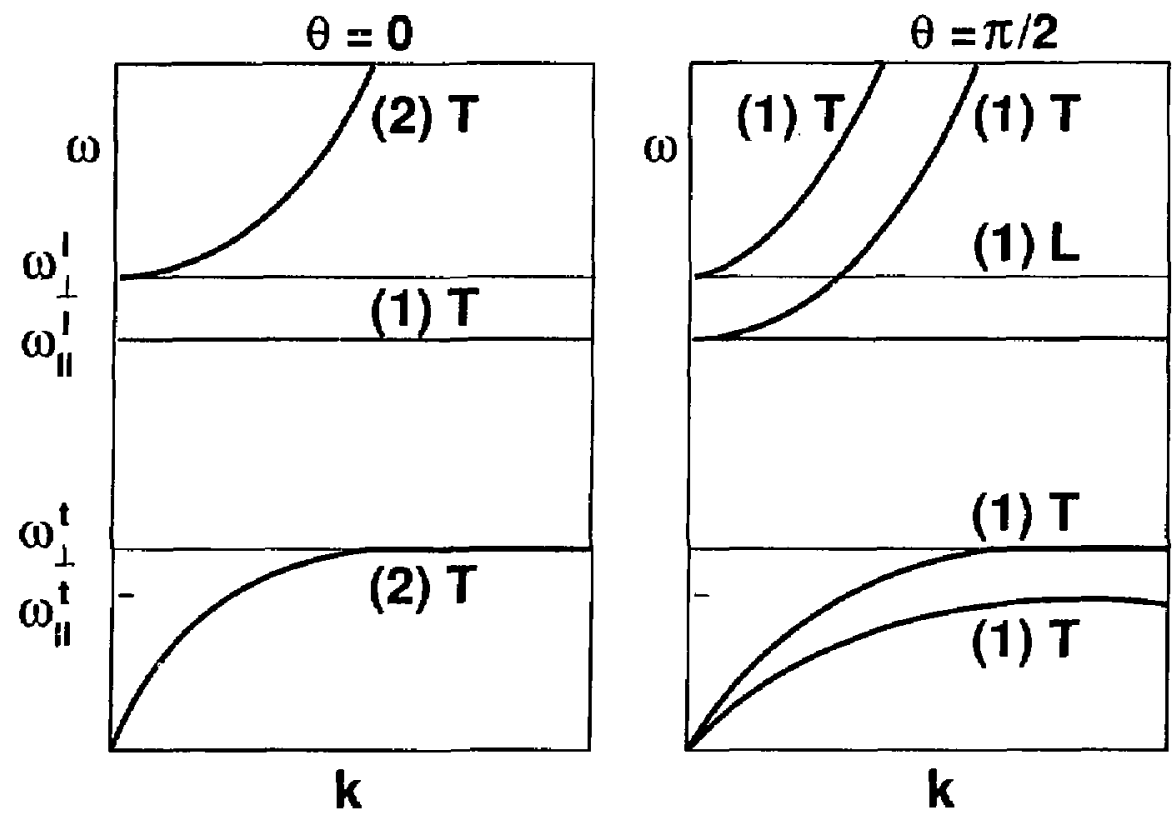

Figure 11. Dispersion relations for a positive uniaxial crystal in the long wavelength region with the electrostatic forces greater than the anisotropic forces. 

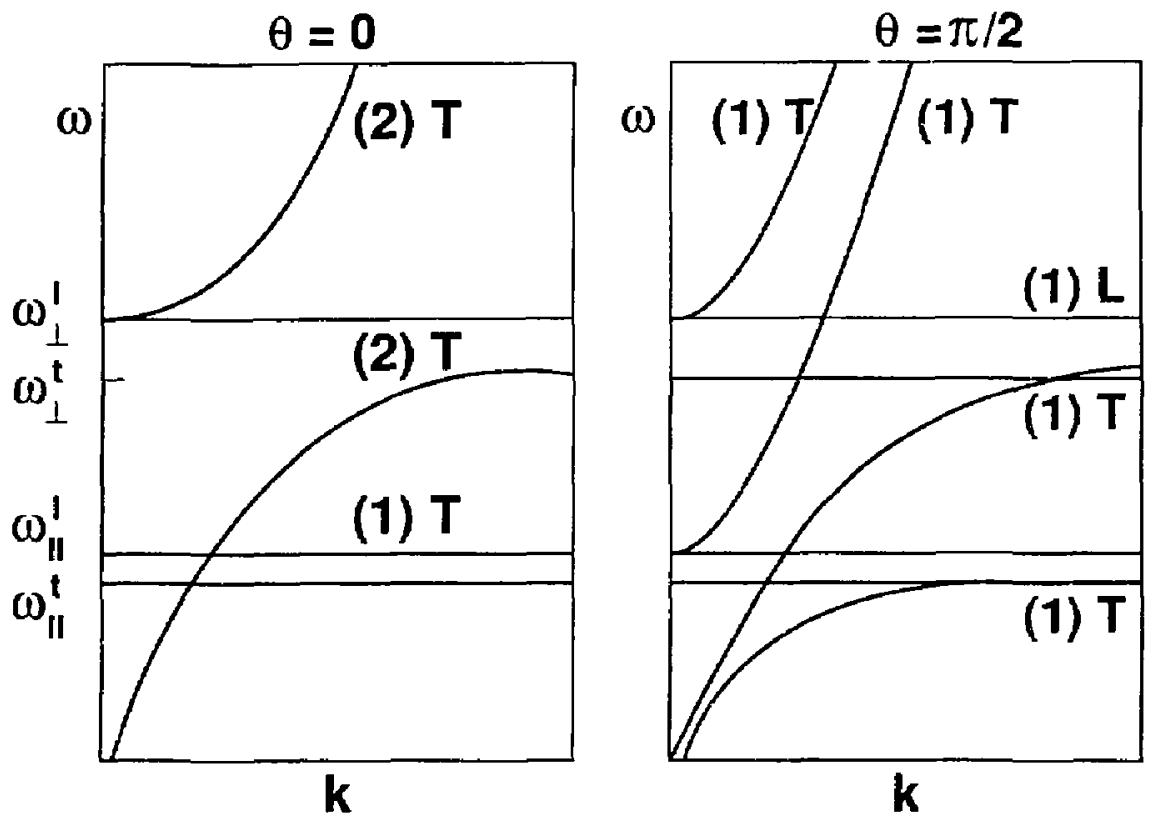

Figure 12. Dispersion relations in the long wavelength region for a positive uniaxial crystal with the anisotropic forces greater than the electrostatic forces. 


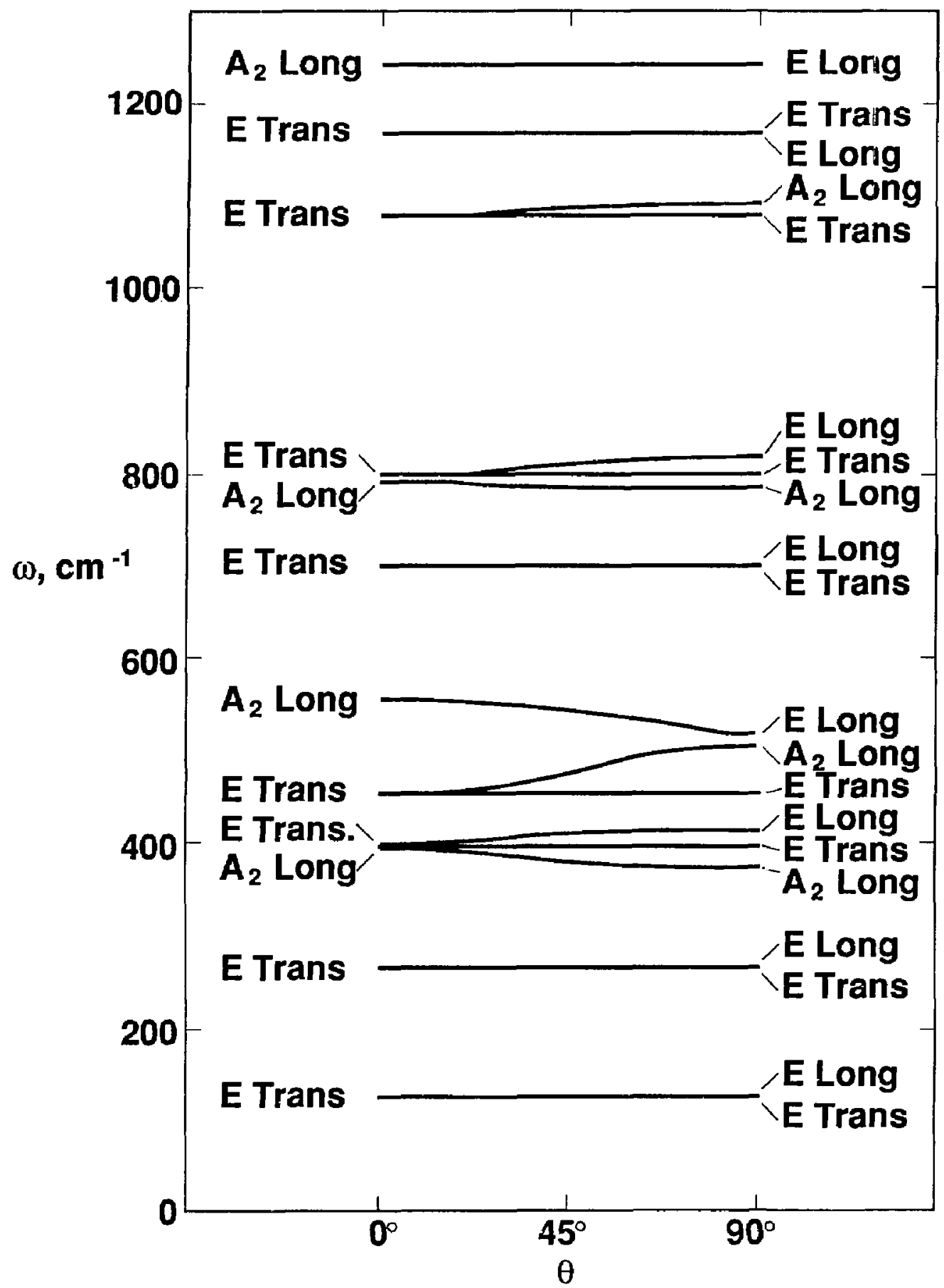

Figure 13. Angular dependence of phonon frequencies in quartz from Ref. 20. 


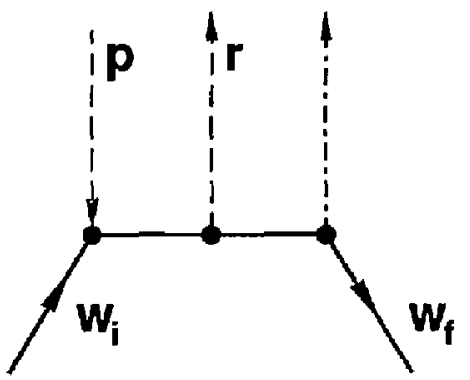

(a)

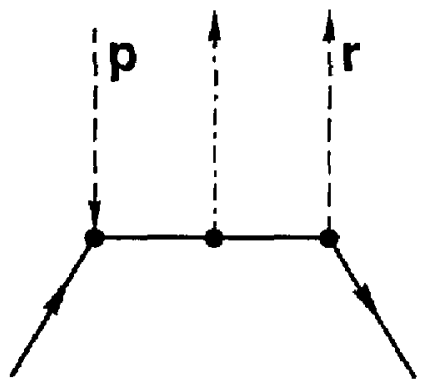

(c)

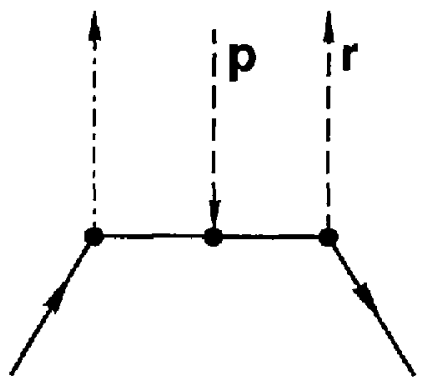

(e)

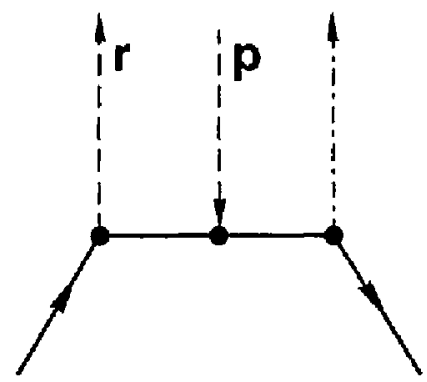

(b)

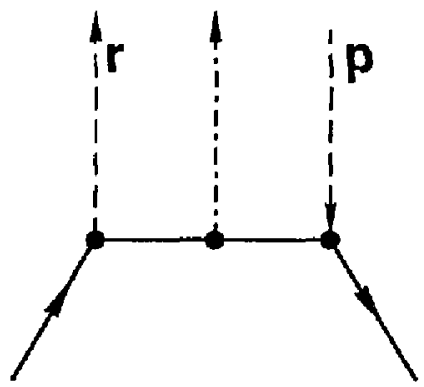

(d)

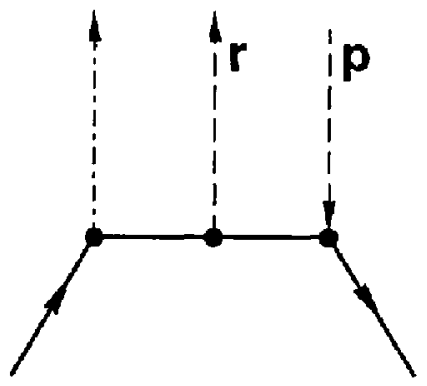

(f)

Figure 14. Feynman diagram of the Raman effect in crystals. 


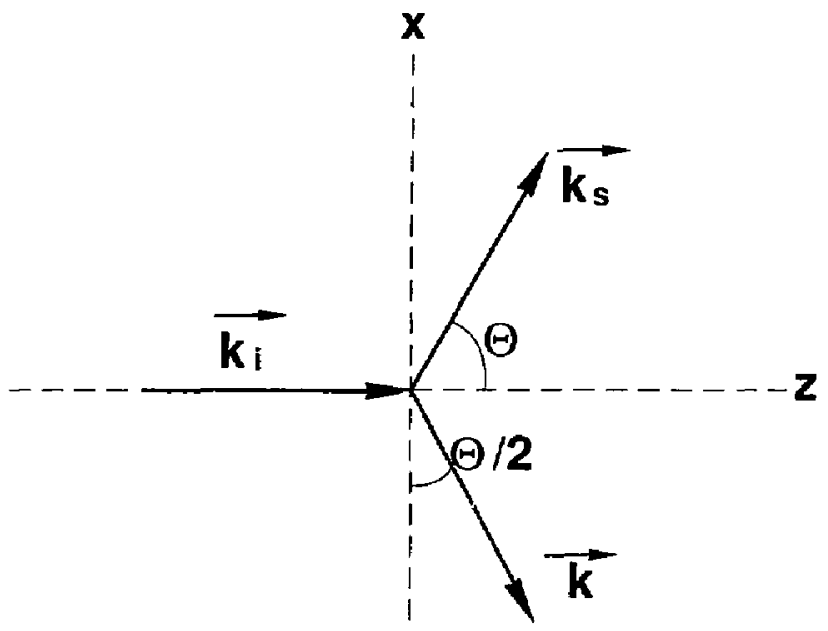

Figure 15. Raman scattering geometry.

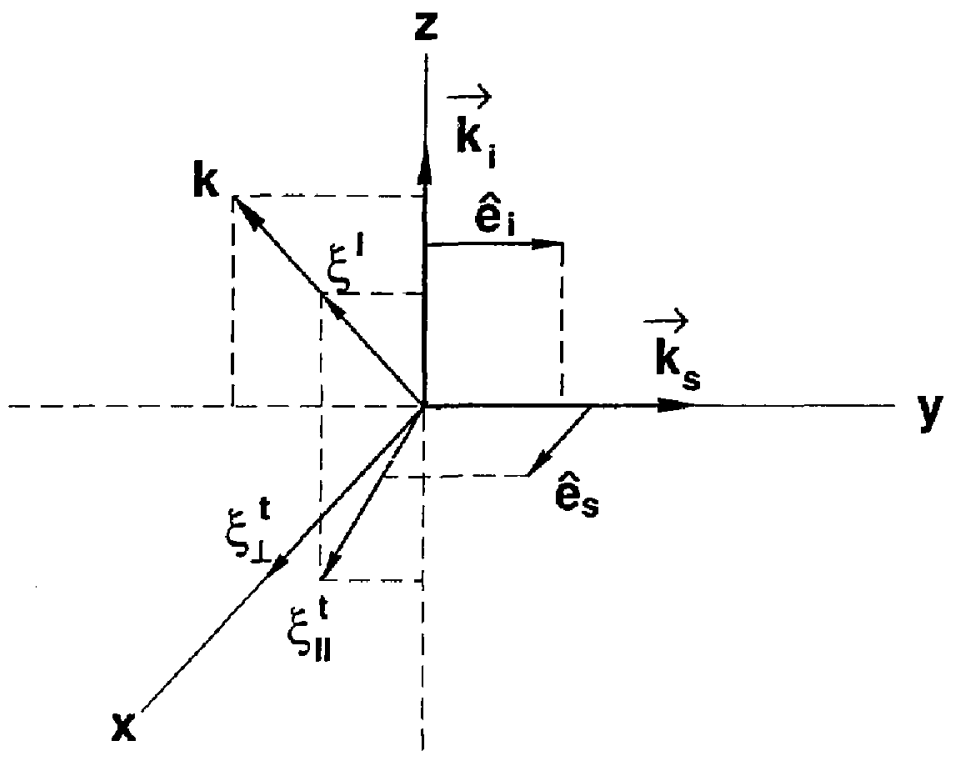

Figure 16. Scattering geometry for measuring the $z(y, x) y$ spectrum. 


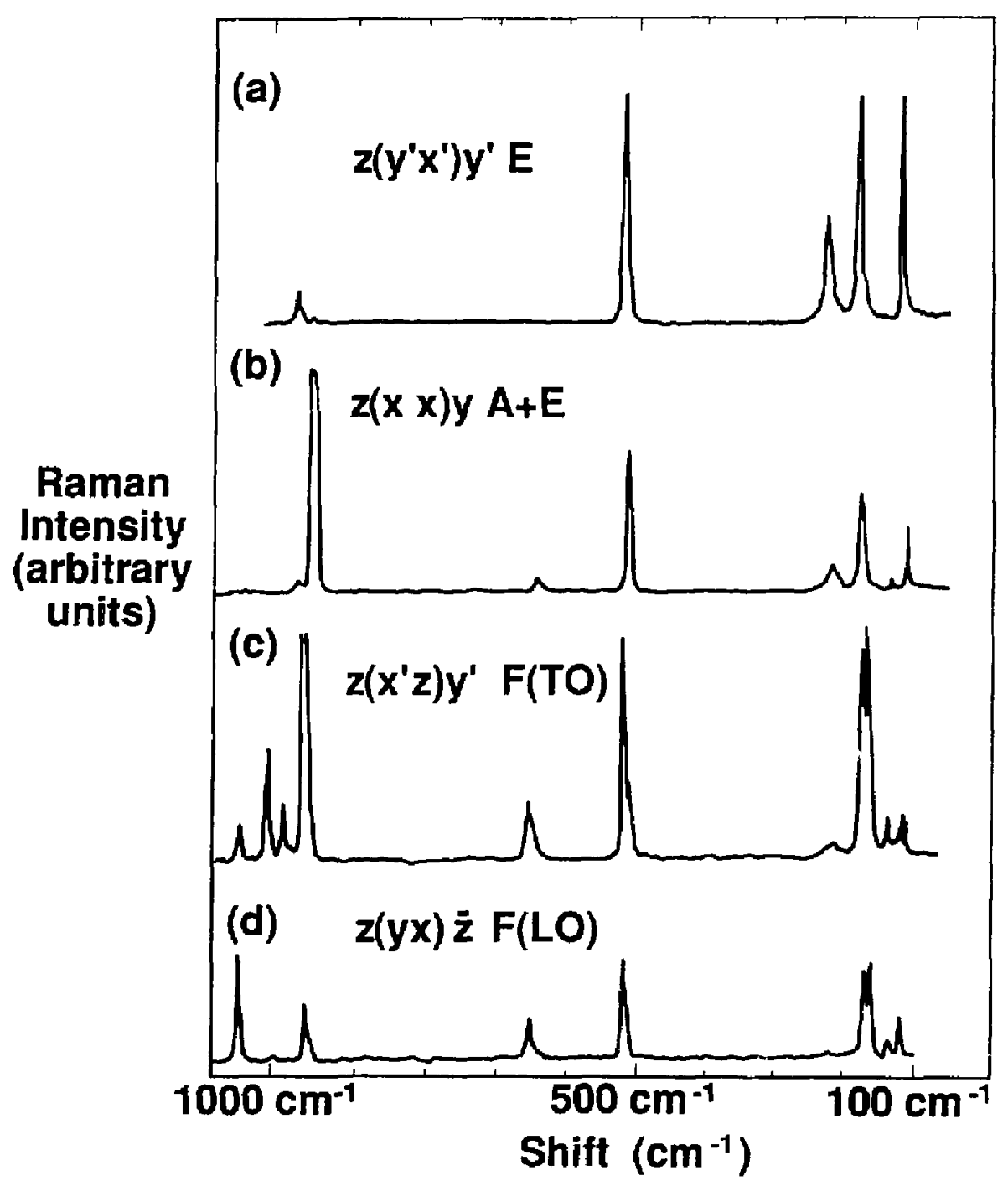

Figure 17. Raman spectra of $\mathrm{NaClO}_{3}$. (a) $z\left(y^{\prime} x^{\prime}\right) y^{\prime}$ spectrum showing $\mathrm{E}$ phonons. (b) $z(x x) y$, corresponding to $A+E$ phonons. (c) $z\left(x^{\prime} z\right) y^{\prime}$, corresponding to $F$ (TO) modes. (d) $z(y x) \bar{z}$ spectrum showing the $F(\mathrm{LO})$ modes. 


\section{Acknowledgments}

The author wishes to thank the following persons for their assistance and discussions during the preparation of this manuscript: Dr. C. Y. She, Dr. A. Holz, Dr. Jorge Nicola, and Dr. Ialo Bonilla.

This manuscript was prepared while the author was a Visiting Professor at the Institute de Fisica, Universidade Federal do Rio Grande do Sul, Porto Algere, Brasil under grants from U.S. Fulbright Commission and the Organization of American States.

\section{References}

1. P. A. Egelstaff, Discuss. Faraday Soc. 43, 149 (1967); B. Chu, Ann. Rev. Phys. Chem. 21, 145 (1970).

2. C. Kittel, Introduction to Solid State Physics (John Wiley and Sons, New York, 1966), 3rd. ed., ch. 5.

3. C. Beck, Anals Acad. Brasil. Ci. 26, 64 (1954).

4. W. Shockley, Electrons and Holes in Semiconductors (Van Nostrand, Co., Inc. New York, 1950).

5. A. Einstein, Amt. Phys. 33, 1275 (1910); L. Brillouin, Ann. de Phys. 17, 88 (1922).

6. H. Z. Cummins, in Quantum Optics, in Proc. of Intern. School of Physics, Varenna, Course 42, edited by R. I. Glauber (Academic Press, New York, 1969).

7. M. Born and K. Huang, Dynamical Theory of Crystal Lattices (University Press, Oxford, 1954).

B. M. A. Heald, Am. J. Phys. 39, 684 (1971).

9. G. B. Benedek, J. B. Lastovka, K. Fritsch, and T. Greytak, J. Opt. Soc. Am. 54, 1284 (1964).

10. K. Huang, Proc. Roy. Soc. A208, 352 (1951).

11. C. Kittel, Quantum Theory of Solids (John Wiley and Sons, New York, 1963).

12. H. Goldstein, Classical Mechanics (Addison-Wesley Publishing Company, Reading, Mass., 1950).

13. R. B. Bames, Zeit. f. Physik 75, 723 (1923).

14. See for example, D. W. Berreman, Phys. Rev. 130, 2193 (1963).

15. J. F. Scott, Am. J. Pliys. 39, 1360 (1971) and the references given there.

16. C. H. Henry and J. J. Hoptield, Phys. Rev. Letters 15, 964 (1965).

17. A. Mooradian and G. B. Wright, Solid State Communications 4, 431 (1966); A. Mooradian and A. L. McWhorter, Phys. Rev. Letters 19, 849 (1967).

18. F. A. Johnson, in Senticonductors, in Proc. of Intern. School of Physics, Varenna, Course 22, edited by R. A. Smith (Academic Press, New York, 1963).

19. T. C. Damon, S. P. S. Porto, and B. Tell, Phys. Rev. 142, 570 (1966).

20. C. Y. She, J. D. Masso, and D. F. Edwards, J. Phys. Chem. Solids 32, 1887 (1971).

21. D. Marcuse, Engineering Quantum Electrodynamics (Harcourt, Brace and World, Inc., New York, 1970).

22. R, Loudon, Proc. Roy. Soc A275, 218 (1963).

23. L. N. Ovander, Optics and Spectroscopy 9, 302 (1960).

24. R. L. Carter, J. Chem. Educ. 48, 297 (1971) and the references given there.

25. C. M. Hartwig, D. L. Rousseau, and S. P. S. Porto, Plyys. Rev. 188, 1328 (1969).

26. R. S. Halford, 1 . Chem. Phys. 14, 8 (1946).

27. D. F. Hornig, /. Chem. Phys. 16, 1063 (1948).

28. V. Henie, Group Theory in Qunntum Mechanics (Clarendon Press, Oxford, 1960).

29. W. G. Fateley, N. T. McDevitt and F. F. Bentley, Appl. Spectro. 25155 (1971).

30. E. B. Wilson, Jr., J. C. Decius, and P. C. Cross, Molecular Vibrations (McGraw-Hill, New York, 1955). ch. 6 .

31. R. Loudon, Adv. in Phys. 13, 423 (1964).

32. H. Poulet, Ann. de Phys., Paris, 10908 (1955).

33. D. F. Edwards and C. Y. She, Am. J. Phys. 401389 (1972).

34. R. Wyckoff, Crysfal Structures, 2nd ed., vol. II (Interscience, New York, 1963).

35. G. Herzberg, Infrared and Raman Spectra (Van Nostrana, Princeton, N.J., 1945).

36. C. Y. She, C. T. Meneely and D. F. Edwards, Colorado State University, Fort Collins, CO, private communication (1973).

37. J. H. Nicola, I. Bonilla, H. Rutt, and D. F. Edwards, Universidade Federal do Rio Grande do Sul, Porto Alegre, Brasil, private communication (1973). 